\title{
Efficacy of Albumin Treatment for Patients with Cirrhosis and Infections Unrelated to Spontaneous Bacterial Peritonitis
}

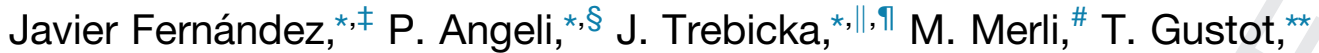

C. Alessandria, ${ }^{\ddagger \neq}$ N. K. Aagaard, ${ }^{\S \S}$ A. de Gottardi, "\|\| T. M. Welzel, " A. Gerbes, "ๆ

G. Soriano, ${ }^{\# \#}$ V. Vargas, ${ }^{\star \star \star}$ A. Albillos, ${ }^{\neq \neq \ddagger}$ F. Salerno, ${ }^{\S \S \S}$ F. Durand,,$"\|\| \|$ R. Bañares, ๆๆๆ

R. Stauber, ${ }^{\# \# \# ~ V . ~ P r a d o, ~}{ }^{\ddagger}$ M. Arteaga, ${ }^{\ddagger}$ M. Hernández-Tejero, ${ }^{\ddagger}$ F. Aziz, ${ }^{\ddagger}$

F. Morando, ${ }^{\S}$ C. Jansen, " B. Lattanzi, ${ }^{*}$ C. Moreno, ${ }^{* \star}$ D. Campion, ${ }^{\ddagger \ddagger}$ H. Gronbaek, ${ }^{\S \S}$

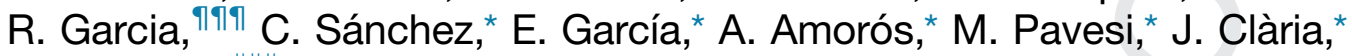

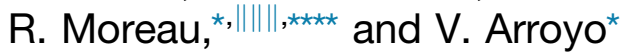

${ }^{*}$ EF Clif, EASL-CLIF Consortium and Grifols Chair, Barcelona, Spain; ${ }^{\ddagger}$ Hospital Clínic, IDIBAPS and CIBERehd, Barcelona, Spain; ' University of Padua, Padua, Italy; "University Hospital of Bonn, Bonn, Germany; "Hospital University of Frankfurt, Frankfurt, Germany; "Sapienza University of Rome, Rome, Italy; **Department of Gastroenterology, Erasme Hospital (ULB), Brussels, Belgium; ${ }^{\ddagger \neq}$ Division of Gastroenterology and Hepatology, Città della Salute e della Scienza Hospital, University of Turin, Turin, Italy; $\$$ Aarhus University Hospital, Aarhus, Denmark; "Ill University of Berne, Berne, Switzerland; "97 University

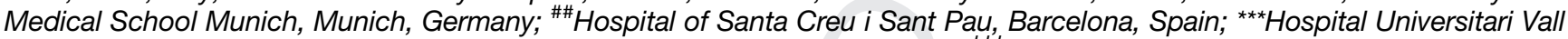
d'Hebron, Universitat Autonoma de Barcelona i ClBERehd, Barcelona, Spain; ${ }^{\neq \neq \neq}$Hospital Universitario Ramon y Cajal, Madrid, Spain; §§§̧ Policlinico IRCCS San Donato, Milan, Italy; "\|ll| Service d'Hépatologie, Hôpital Beaujon, Assistance Publique-Hôpitaux de Paris, Clichy, France; ${ }^{979}$ Hospital Gregorio Marañon, Madrid, Spain; \#\#\# Medical University of Graz, Graz, Austria; and ${ }_{* * \star *}^{*}$ Inserm, Université Paris Diderot-Paris 7, CNRS, Centre de Recherche sur I'Inflammation, Paris, France

BACKGROUND \& AIMS:

We performed a randomized trial to determine whether albumin should be administered to patients with infections unrelated to spontaneous bacterial peritonitis (SBP).

METHODS:

We performed a multicenter, open-label trial in which 118 patients with cirrhosis, non-SBP infections, and additional risk factors for poor outcome were randomly assigned to receive antibiotics plus albumin (study group; $n=61$ ) or antibiotics alone (control group; $n=57$ ). The primary outcome was in-hospital mortality; secondary outcomes were effect of albumin on disease course.

RESULTS:

There were no significant differences at baseline between groups in results from standard laboratory tests, serum markers of inflammation, circulatory dysfunction, or liver severity scores. However, the combined prevalence of acute on chronic liver failure (ACLF) and kidney dysfunction was significantly higher in the study group $(44.3 \%$ vs $24.6 \%$ in the control group; $P=.02)$, indicating greater baseline overall severity. There was no significant difference in the primary outcome between groups $(13.1 \%$ in the study group vs $10.5 \%$ in the control group; $P=$ .66). Circulatory and renal functions improved in only the study group. A significantly higher proportion of patients in the study group had resolution of ACLF $(82.3 \%$ vs $33.3 \%$ in the control group; $P=.03$ ). A significantly lower proportion of patients in the study group developed nosocomial infections (6.6\% vs $24.6 \%$ in the control group; $P=.007)$.

CONCLUSIONS:

In a randomized trial of patients with advanced cirrhosis and non-SBP infections, in-hospital mortality was similar between those who received albumin plus antibiotics vs those who received only antibiotics (controls). However, patients given albumin were sicker at baseline and, during the follow-up period, a higher proportion had ACLF resolution and a lower proportion had nosocomial infections. ClinicalTrials.gov no: NCT02034279. 
The transition from compensated to decompensated cirrhosis occurs in the setting of an activation of the immune system, chronic systemic inflammation, and multiorgan dysfunction. ${ }^{1-4}$ Systemic inflammation induces an immune-modulatory response that increases the risk of infection. ${ }^{5}$ When infection develops in decompensated cirrhosis, there is a secondary burst of inflammation, ${ }^{1-5}$ which may lead to the development of acute-on-chronic liver failure (ACLF).

The pioneer randomized controlled trial (RCT) ${ }^{6}$ done in patients with spontaneous bacterial peritonitis (SBP) showing that intravenous albumin reduced the prevalence of type- 1 hepatorenal syndrome (HRS) and hospital mortality, introduced the prophylactic use of albumin in patients with bacterial infections. However, 2 subsequent negative RCTs in patients with non-SBP infections suggested that the beneficial effects of albumin did not extend to all types of infections. ${ }^{7,8}$ However, short-term mortality rates were very low in both studies (5\%).

We performed a retrospective analysis of 3 cohorts to identify patients with cirrhosis and non-SBP infections at higher risk of hospital mortality. ${ }^{9}$ Mortality was highly dependent of the type of infection: high in endocarditis and secondary peritonitis, intermediate in pneumonia and bacteremia, and low in urinary tract infection (UTI) and cellulitis. Hospital mortality rate of each type of infection was significantly higher in patients with poor liver/renal function.

Based on these data, we designed an RCT aimed to assess if albumin treatment impacted hospital survival among patients with cirrhosis with non-SBP infections at high risk of hospital mortality. The secondary endpoint was the effect of albumin treatment on patients' clinical course during hospitalization.

\section{Patients and Methods}

\section{Study Oversight}

The INFECIR-2 Albumin Prevention study (Albumin administration in the prevention of HRS and death in patients with cirrhosis, bacterial infections other than SBP and high risk of hospital mortality; ClinicalTrials. gov: NCT02034279) is an EASL-CLIF Consortium investigator-initiated, phase IV, randomized, openlabel, parallel, multicenter European trial promoted by the Fundació Clínic (Hospital Clínic, University of Barcelona, Barcelona, Spain). Written informed consent was obtained from all patients. All authors had access to the study data, critically reviewed the manuscript, and approved the final draft. The trial was performed in 27 centers, in accordance with the Declaration of Helsinki, and was approved by the different local ethics committees and competent authorities.

\section{What You Need to Know}

Background

We performed a randomized trial to determine whether albumin should be administered to patients with infections unrelated to spontaneous bacterial peritonitis (SBP).

\section{Findings}

Among patients with advanced cirrhosis and non-SBP infections, in-hospital mortality is similar between those who receive the combination of albumin and antibiotics and those who received only antibiotics. However, a higher proportion of patients given albumin have resolution of acute on chronic liver failure and a lower proportion develop nosocomial infections.

\section{Implications for Patient Care}

Patients with advanced cirrhosis and non-SBP infections who receive the combination of albumin and antibiotics have better outcomes than patients who received only antibiotics.

\section{Patients}

Inclusion criteria. Patients with non-SBP infections were screened for eligibility. Inclusion criteria were: age $\geq 18$ years; liver cirrhosis; UTI, pneumonia, spontaneous/secondary bacteremia, cellulitis, acute cholangitis, or suspected bacterial infection; and advanced liver disease (serum creatinine $\geq 1.2 \mathrm{mg} / \mathrm{dL}$, serum sodium $\leq 130 \mathrm{mEq} / \mathrm{L}$, and/or serum bilirubin $\geq 4 \mathrm{mg} / \mathrm{dL}$ ). Patients with pneumonia or spontaneous/secondary bacteremia required the presence of at least 1 of these criteria (serum creatinine $\geq 1.2 \mathrm{mg} / \mathrm{dL}$, serum sodium $\leq 130 \mathrm{mEq} / \mathrm{L}$, or serum bilirubin $\geq 4 \mathrm{mg} / \mathrm{dL})^{9}$; in the rest, at least 2 were required. Patients with UTI or suspected infections required additionally at least 1 diagnostic criterion of systemic inflammatory response syndrome (temperature $>38^{\circ} \mathrm{C}$ or $<36^{\circ} \mathrm{C}$; heart rate $>90$ beats/ minute; respiratory rate $>20$ breaths/minute; white blood cell (WBC) count $>12.000$ or $<4000 / \mathrm{mm}^{3}$ ); and a serum $C$ reactive protein (CRP) level $\geq 1 \mathrm{mg} / \mathrm{dL}$.

Exclusion criteria. The main exclusion criteria were: infections evolving for $>72$ hours, septic shock, endocarditis, severe acute respiratory distress syndrome $\left(\mathrm{PaO}_{2} / \mathrm{FIO}_{2} \leq 100\right)$, active/recent variceal bleeding (unless controlled for $>48$ hours), type- 1 HRS (IAC criteria), Q6 ACLF grade-3, malignancy (except for hepatocellular carcinoma within Milan criteria or nonmelanocytic skin cancer), chronic heart failure (New York Heart Association functional class II-IV), severe chronic pulmonary disease (Global Initiative for Chronic Obstructive Lung Disease IV), liver transplantation, human immunodeficiency virus infection, contraindications to albumin, 
albumin administration $(\geq 80 \mathrm{~g})$ in the last 2 days, and SBP coinfection. All exclusion criteria are detailed in the Supplementary Material.

\section{Randomization}

Randomization was performed in blocks of 4 (1:1 ratio). It was centralized at the Data Management Center of the European Foundation for the Study of Chronic Liver Failure, was independent for each center, and performed online. A computer-generated randomization list (block sizes blinded for the investigators) was used for treatment allocation. Blocks were stratified by the type (pneumonia vs other infections) and the site of acquisition of infection (nosocomial vs other).

\section{Treatment Allocation, Study Protocol, and Follow-Up}

Eligible patients were randomized to receive antibiotics alone (control group) or antibiotics plus albumin (study group). Suggestions for the choice of empirical antibiotics were given in the study protocol (Supplementary Material). The study albumin (Albutein $20 \%$, Instituto Grifols) was administered at a dose of 1.5 $\mathrm{g} / \mathrm{kg}$ body weight at Day 1 and $1 \mathrm{~g} / \mathrm{kg}$ body weight at Day 3 up to a maximum of $150 \mathrm{~g}$ and $100 \mathrm{~g}$, respectively, in patients with body weight $>100 \mathrm{~kg}$, and a minimum of $90 \mathrm{~g}$ and $60 \mathrm{~g}$, respectively, in patients with body weight $<60 \mathrm{~kg}$. Albumin administration was started within the first 8 hours after randomization and infused in 6-12 hours with close clinical monitoring to prevent volume overload. Follow-up visits were performed on Days 3 and 7 , and weekly thereafter until infection resolution. Data on liver transplantation, hospital and 90-day mortality, and causes of death were recorded within 3 months after enrollment. No other doses of albumin were allowed in both treatment arms throughout the hospitalization unless needed for the treatment of type-1 HRS, total paracentesis, or SBP.

As per protocol, routine laboratory tests, serum albumin levels, and plasma concentration of interleukin 6 (IL6) and renin (PRC) were measured at Days 1 and 3 (before albumin doses in the group) and at infection resolution or Day 7. PRC and IL6 were measured by chemiluminescent immunoassay and enzyme-linked immunosorbent assay, respectively.

\section{Assessment of Disease Severity at Enrollment and Definitions}

We enrolled patients with prespecified criteria for advanced cirrhosis. ${ }^{10}$ Disease severity at enrollment was estimated by standard tests, Child-Pugh, Model for EndStage Liver Disease, and CLIF-Consortium organ failure scores ${ }^{11}$ (Supplementary Table 1 ) and by the presence of ACLF or kidney dysfunction (KD). Reasons for considering these 2 later variables to assess disease severity are described in the Supplementary Material and in Supplementary Tables 2 and 3.

Definitions and diagnostic criteria of ACLF and bacterial infections are also detailed in the Supplementary Material.

\section{Study Outcomes}

The prespecified primary outcome was mortality in hospital. Secondary outcomes were: the grade of circulatory dysfunction (as estimated by PRC), systemic inflammation (as estimated by plasma concentration of IL6 and other biomarkers) and complications related with these abnormalities (ACLF and nosocomial bacterial infections), and 90-day mortality. We also sought to identify predictors of in-hospital and 90-day mortality, and development of ACLF, bacterial infection, and pulmonary edema during hospitalization.

\section{Statistical Analysis}

Unless specified, analyses were performed on an intention-to-treat (ITT) basis. In-hospital mortality was estimated to be about $29 \%$ in patients who did not receive albumin. Based on this assumption, a global sample size of 512 patients (256 per treatment arm) was estimated to detect an absolute reduction of $11 \%(18 \%$ mortality rate) in patients treated with albumin $(80 \%$ statistical power). ${ }^{6,9}$ A 2 -way $5 \%$ type-I error and a $10 \%$ drop-out rate was assumed.

Statistical analysis was performed using unpaired Student $t$ test for continuous variables with parametric distribution, Mann-Whitney $U$ test for those with nonparametric distribution, and chi-square test for qualitative variables, applying Yate correction when required. Differences were considered significant at the level 0.05. Survival was analyzed by Kaplan-Meier method and compared between groups with log-rank test. Risk factors were assessed using univariate analysis, and variables that were significantly associated with the different outcome events were included in the multivariate analysis by fitting logistic regression models to obtain adjusted treatment estimates and independent risk factors (forward stepwise selection method). Competing risk analyses were used to evaluate the impact of albumin treatment on the primary outcome, estimating the cumulative incidence of death at hospital discharge, whereas liver transplantation was treated as a competing event. Competing risk analysis was also used to estimate the cumulative incidence of new proven bacterial infection during hospitalization in both treatment arms. Liver transplantation was treated again as a competing event. The estimated cumulative incidences were compared by means of the Gray test. Analysis was done with SPSS version 18.0 (IBM, Armonk, NY) and SAS version 9.1 (SAS Institute, Cary, NC). 
Table 1. Characteristics of the Patients at Baseline

$58.3 \pm 13.7$
$35(58.3)$
$31(50.8)$
$21(35.0)$
$48(80.0)$
$28(46.7)$

$78.2 \pm 11.7$

$5.9(6.2)$

$\mathrm{mg}$

Median value (interquartile range) for serum bilirubin, $\mathrm{mg} / \mathrm{dL}$

Serum albumin, $g / L$

Prothrombin ratio, \%

Serum creatinine, $\mathrm{mg} / \mathrm{dL}$

Serum sodium, $m E q / L$

Serum bilirubin $\geq 4 \mathrm{mg} / \mathrm{dL}, \%$

Serum creatinine $\geq 1.2$ $\mathrm{mg} / \mathrm{dL}, \%$

Serum sodium $<130$ $\mathrm{mEq} / \mathrm{L}, \%$

Serum creatinine $\geq 1.2$ $\mathrm{mg} / \mathrm{dL}$ or serum sodium $<130$ $\mathrm{mEq} / \mathrm{L}, \%$

Median values (interquartile range) for markers of systemic inflammation and circulatory dysfunction White blood cell count, $\times 10^{9} / \mathrm{L}$

Serum C-reactive protein, $\mathrm{mg} / \mathrm{dL}$

Plasma interleukin 6, $\mathrm{pg} / \mathrm{mL}$

Plasma renin concentration, micro IU/mL

Presence of ACLF or kidney dysfunction, n (\%)

ACLF-1

ACLF-2

ACLF

Kidney dysfunction

ACLF or kidney dysfunction

Liver scores

Child-Pugh score, points

$23.6 \pm 9.1$

$1.27 \pm 0.66$

$33(55.9)$

32 (53.3)

35 (57.4)

$50(82.0)$

37 (21-155)

13 (21.3)

$4(6.6)$

$10(16.4)$

27 (44.3)

$10.4 \pm 9.8$

\begin{tabular}{ccc} 
Albumin plus & Antibiotics & \\
antibiotics & alone & $P$ \\
$(n=61)$ & $(n=57)$ & value \\
\hline
\end{tabular}

$58.5+11.0$

$39(66.1)$

$39(68.4)$

17 (28.8)

$42(71.2)$

$27(45.8)$

$78.4 \pm 11.7$

$7.3(6.8)$

$24.0 \pm 8.4$

$48.9 \pm 16.5$

$1.13 \pm 0.38$

$132.8 \pm 5.4$

36 (63.2)

28 (49.1)

26 (45.6)

44 (77.2)

6.9 (4.9-10.1)

$15.8(6.4-42.1)$

$12.7(4.6-46.5)$

41 (24-108)

125 (34-399)

407

408

409

410

411

412

413

414

415

416

417

418

.26

.92

419

420

421

.94

.25

422

423

424

425

426

427

428

429

430

431

432

433

434

435

436

437

438

439

440

441

442

443

444

445

446

447

448

449

450

451

452

453

8 (14.0)

$1(1.8)$

9 (15.8)

5 (8.8)

14 (24.6)

.63

454

455

456

.21

.025

457

458

459

$10.5 \pm 10.0$

.77

$19.8 \pm 5.1$ 


\begin{tabular}{|c|c|c|c|}
\hline Characteristics & $\begin{array}{l}\text { Albumin plus } \\
\text { antibiotics } \\
(\mathrm{n}=61)\end{array}$ & $\begin{array}{l}\text { Antibiotics } \\
\text { alone } \\
(\mathrm{n}=57)\end{array}$ & $\begin{array}{c}P \\
\text { value }\end{array}$ \\
\hline \multicolumn{4}{|l|}{$\begin{array}{l}\text { Model of End-stage } \\
\text { Liver Disease score, } \\
\text { points }\end{array}$} \\
\hline $\begin{array}{l}\text { CLIF-Consortium organ } \\
\text { failure score, points }\end{array}$ & $7.4 \pm 1.7$ & $7.5 \pm 1.6$ & .77 \\
\hline \multicolumn{4}{|l|}{$\begin{array}{l}\text { Site and severity of } \\
\text { infection, } \mathrm{n}(\%)\end{array}$} \\
\hline Pneumonia & $17(27.9)$ & $22(38.6)$ & .22 \\
\hline $\begin{array}{l}\text { Spontaneous or } \\
\text { secondary } \\
\text { bacteremia }\end{array}$ & $15(24.6)$ & $11(19.3)$ & .49 \\
\hline Urinary tract infection & $12(19.7)$ & $8(14.0)$ & .41 \\
\hline Cellulitis & $2(3.3)$ & $6(10.5)$ & .15 \\
\hline Cholangitis & $3(4.9)$ & 0 & .24 \\
\hline Unproven infections & $10(16.4)$ & $9(15.8)$ & .93 \\
\hline Other ${ }^{2}$ & $2(3.2)$ & $1(1.8)$ & 1.0 \\
\hline Presence of sepsis & $35(57.4)$ & $28(49.1)$ & .37 \\
\hline \multicolumn{4}{|l|}{$\begin{array}{l}\text { Site of acquisition of } \\
\text { infection, } \mathrm{n}(\%)\end{array}$} \\
\hline Community-acquired & $27(45.8)$ & $25(42.4)$ & .78 \\
\hline Health care-associated & $14(23.3)$ & $15(25.0)$ & \\
\hline Nosocomial & $18(30.0)$ & $19(31.7)$ & \\
\hline \multicolumn{4}{|l|}{$\begin{array}{l}\text { Results of microbial } \\
\text { culture, } \mathrm{n}(\%)\end{array}$} \\
\hline Culture-positive & $31(50.8)$ & $32(56.1)$ & .56 \\
\hline $\begin{array}{l}\text { Presence of multidrug- } \\
\text { resistant bacteria }\end{array}$ & $10(16.4)$ & $14(24.6)$ & .27 \\
\hline
\end{tabular}

NOTE. Plus-minus values are means $\pm \mathrm{SD}$. Analyses were performed on an intention-to-treat basis.

ACLF, acute-on-chronic liver failure.

${ }^{a}$ One cholecystitis and 1 Clostridium difficile infection occurred in the albumin-plus-antibiotics group, and 1 spontaneous bacterial empyema in the antibioticsalone group.

\section{Results}

\section{Baseline Characteristics}

Patients' enrollment. The study was started on September 1, 2014, and finished in December 31, 2016. Overall, of the 534 patients who were screened from September 2014 to April 2016, 433 were excluded (inclusion rate, 19\%). Thereafter, investigators were instructed to record only those patients included. Finally, a total of 136 patients were enrolled and 18 were considered inclusion errors (Supplementary Figure 1). The final ITT population consisted of 118 patients, 61 in the study group and 57 in the control group.

Demographic characteristics and clinical data. The prevalence of alcoholic cirrhosis was higher in the control group (68.4\% vs $50.8 \%$ in the study group; $P=.05$ ) (Table 1). There were no differences in other demographic or clinical data.

Severity of cirrhosis and systemic inflammation. There were no significant differences between groups in standard liver and renal function tests and liver scores (Table 1). However, the combined prevalence of ACLF and $\mathrm{KD}$ was significantly higher in the study group
(44.3\% vs $24.6 \% ; P=.025$ ), suggesting significantly higher disease severity.

The grade of circulatory dysfunction (PRC) was also similar between groups as well as the severity of systemic inflammation, as estimated by WBC count, plasma IL6 concentrations, and serum CRP levels.

Type, severity, and microbiology of bacterial infections. Median time from diagnosis of infection to study inclusion was 1 day in both groups. Pneumonia, bacteremia, and UTI were the most frequent infections. Sixtythree patients showed signs of sepsis. Infections were community acquired in 52 patients (Table 1). Half of the infections were associated with positive cultures (24 were caused by multiresistant bacteria). There were no significant differences between groups in the type, site of acquisition, severity, and microbiology of infections (Supplementary Material and Supplementary Table 4).

\section{Albumin Administration}

All except 5 patients in the study group received the scheduled doses of $20 \%$ albumin on Days 1 and 3. Four patients did not receive albumin on Day 3 because of pulmonary edema $(n=3)$ or septic shock $(n=1)$.
525

526

527

528

529

530

531

532

533

534

535

536

537

538

539

540

541

542

543

544

545

546

547

548

549

550

551

552

553

554

555

556

557

558

559

560

561

562

563

564

565

566

567

568

569

570

571

572 
Table 2. Effects of Treatment on Standard Laboratory Tests, Biomarkers of Systemic Inflammation and Circulatory Dysfunction, and the Course of Bacterial Infection and ACLF Diagnosed at Enrollment

\begin{tabular}{cccccc}
\hline & $\begin{array}{c}\text { Albumin plus } \\
\text { antibiotics } \\
\text { Variable }\end{array}$ & $\begin{array}{c}P \text { value for } \\
\text { within-group } \\
\text { comparison }^{a}\end{array}$ & $\begin{array}{c}\text { Antibiotics } \\
\text { alone } \\
(\mathrm{n}=51)\end{array}$ & $\begin{array}{c}P \text { value for } \\
\text { within-group } \\
\text { comparison }^{b}\end{array}$ & $\begin{array}{c}P \text { value for } \\
\text { between-group } \\
\text { comparison }^{c}\end{array}$ \\
\hline
\end{tabular}

Serum albumin and liver, kidney, and circulatory function

Serum albumin, $g / L$ within-group

$(n=57)$ comparison $^{b}$

Baseline

Day 3-7

Median value for serum bilirubin (IQR), $m g / d L$

Baseline

Day 3-7

$25.9 \pm 6.4$
$31.2 \pm 6.5$

$26.0 \pm 5.7$

Prothrombin ratio, \% Baseline Day 3-7 Baseline Day 3-7

Serum sodium, $m E q / L$ Baseline Day 3-7

Mean arterial pressure, $\mathrm{mm} \mathrm{Hg}$ Baseline Day 3-7

Median value for plasma renin concentration (IQR), $\mu / \mathrm{U} / \mathrm{m}^{d}$ Baseline Day 3-7

Median value for markers of inflammation (IQR)

White blood cell count, $\times 10^{9} / \mathrm{L}$ Baseline Day 3-7

Serum C-reactive protein, $\mathrm{mg} / \mathrm{dL}$

Baseline Day 3-7

Plasma interleukin 6, pg/mL ${ }^{e}$ Baseline Day 3-7

Course of infection, $\mathrm{n}(\%)$

Adequate empirical antibiotics Septic shock Infection resolution

Course of ACLF or kidney dysfunction, n/N (\%)

Resolution of $\mathrm{ACLF}^{f}$

Resolution of kidney dysfunction

Resolution of ACLF or kidney dysfunction
Serum creatinine, $m g / d L$
$<.0001$

$4.3(1.7-7.8)$
$4.0(2.3-8.7)$

$49.6 \pm 13.0$

$48.1 \pm 14.2$

$1.3 \pm 0.7$

$1.1 \pm 0.6$

$131.2 \pm 6.2$

$134.9 \pm 5.7$

$78.1 \pm 11.7$

$82.1 \pm 11.8$

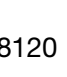

$5.1(2.1-11.0)$

4.3 (1.7-9.7)

.5414

$<.001$

$48.9 \pm 16.5$

$49.5 \pm 16.1$

.3793

$1.1 \pm 0.4$

$1.1 \pm 0.4$

.0116

$132.7 \pm 5.4$

$133.5 \pm 5.1$

$<.0001$

$79.1+11.0$

.0167

$80.1 \pm 10.5$

.4758

125 (34-399)

242 (46-903)

161 (18-393)

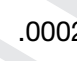

91 (25-768)

.4426

$6.9(4.9-10.1)$

$7.2(4.8-10.5)$

5.8 (3.9-9.0)

.0003

$6.2(4.3-10.0)$

.1347

$15.8(6.4-42.1)$

$12.7(4.6-46.5)$

8.7 (3.0-28.0)

$<.0001$

$11.5(3.8-33.4)$

$<.0001$

.81

37 (21-155)

32 (18-69)

41 (24-108)

32 (17-72)

.0322

51 (89.5)

55 (90.2)

$6(9.8)$

53 (89.8)

$\begin{array}{lc}- & 51(89.5) \\ - & 2(3.5) \\ - & 54(96.4)\end{array}$

.2900

.71

14/17 (82.3)

$7 / 10(70.0)$

$21 / 27(77.8)$
-

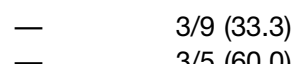

- $\quad 3 / 5(60.0)$

- $\quad 6 / 14(42.9)$
649

650

651

652

653

654

655

656

657

658

659

660

661

662

663

664

665

666

667

668

669

670

671

672

673

674

675

676

677

678

679

680

681

682

683

684

685

686

ACLF, acute-on-chronic liver failure; IQR, interquartile range.

${ }^{a}$ Comparison of baseline vs Day 3-7, in the albumin-plus-antibiotics group.

${ }^{b}$ Comparison of baseline vs Day 3-7, in the antibiotics-alone group.

${ }^{\circ}$ Between group comparison was performed at Day 3-7.

${ }^{d}$ Plasma renin concentrations were determined at Day 1, and Day 3-7 in 40 patients in the albumin-plus-antibiotics group and in 41 patients in the antibiotics-alone group.

Interleukin 6 levels were determined at Day 1, and Day 3-7 in 48 patients in the albumin-plus-antibiotics group and in 47 patients in the antibiotics-alone group.

${ }^{t}$ The 4 patients with ACLF-2 in the albumin-plus-antibiotics group solved the syndrome; this did not occur in the single patient with ACLF-2 of the antibiotics-alone group.
687

688

689

690

691

692

693

694

695

696 


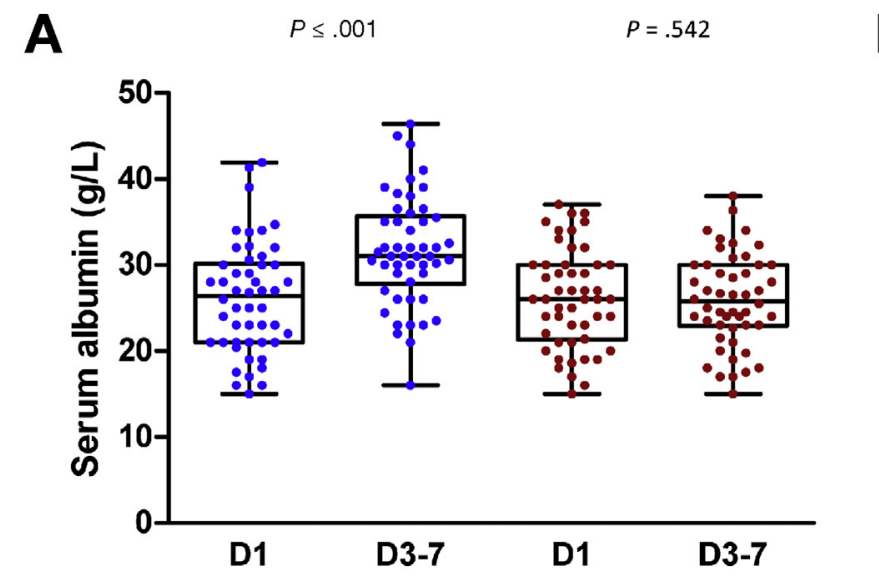

B $\quad P \leq .00$

$P=.443$
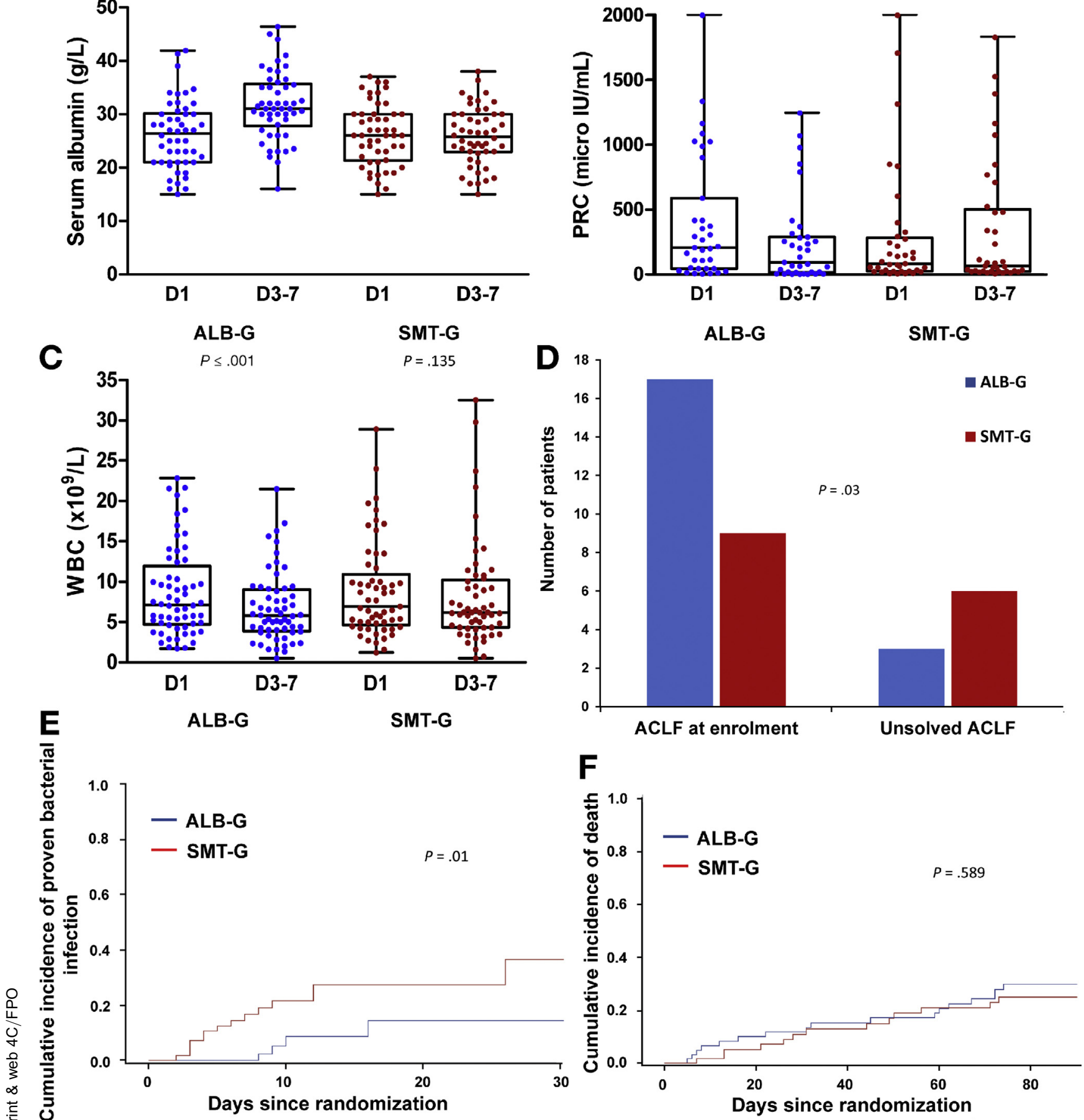

Figure 1. Albumin therapy in patients with advanced cirrhosis and non-SBP infections induces multifaceted beneficial effects but no increase in survival. $(A)$ Individual changes in serum albumin concentration during treatment. Serum albumin concentration increased significantly only in patients receiving albumin; $P<.001$ for the between-group comparison. $(B)$ Individual changes in PRC during treatment. Albumin treatment was associated with a marked reduction in PRC; $P=.004$ for the between-group comparison. $(C)$ Individual changes in WBC during treatment. WBC decreased in both groups, but differences were statistically significant only in patients receiving albumin; $P$ value not significant $(0.14)$ for the between-group comparison. (D) Clinical course of ACLF diagnosed at enrollment. It resolved more frequently in patients receiving albumin. (E) Cumulative incidence of new proven bacterial infections was significantly higher in the control group. $(F)$ Cumulative incidence of death was similar between groups. Liver transplantation was considered as a competing risk of bacterial infection development and death. In every panel, blue color represents patients treated with albumin and antibiotics, and red color those treated with antibiotics alone. ALB-G; PRC; SMT-G. 
Table 3. New Events During Hospitalization and Short-Term Mortality

\begin{tabular}{|c|c|c|c|}
\hline Event & $\begin{array}{l}\text { Albumin plus antibiotics } \\
\qquad(\mathrm{n}=61)\end{array}$ & $\begin{array}{l}\text { Antibiotics alone } \\
\quad(n=57)\end{array}$ & $P$ value \\
\hline \multicolumn{4}{|l|}{ New bacterial infections } \\
\hline \multicolumn{4}{|l|}{ Patients with infections, n (\%) } \\
\hline Patients with proven or unproven infections, n (\%) & $6(9.8)$ & $14(24.6)$ & .03 \\
\hline Patients with proven infections, $\mathrm{n}(\%)$ & $4(6.6)$ & $14(24.6)$ & .007 \\
\hline Type and severity of infections, $n(\%)$ & & & .27 \\
\hline Pneumonia & $1(1.6)$ & $4(7.0)$ & \\
\hline Spontaneous or secondary bacteremia & $1(1.6)$ & $4(7.0)$ & \\
\hline Urinary tract infection & $1(1.6)$ & $3(5.3)$ & \\
\hline Spontaneous bacterial peritonitis & $1(1.6)$ & 0 & \\
\hline Cellulitis & $1(1.6)$ & $2(3.5)$ & \\
\hline Unproven infections & $2(3.3)$ & 0 & \\
\hline Clostridium difficile infection & 0 & $2(3.5)$ & \\
\hline Other & 0 & $1(1.8)$ & \\
\hline Number of infectious episodes & 7 & 16 & \\
\hline Presence of sepsis & $4(6.6)$ & $6(10.5)$ & .44 \\
\hline \multicolumn{4}{|l|}{$\begin{array}{l}\text { Microbiology and course of infection, } \\
\mathrm{n} \text { (\% of infections) }\end{array}$} \\
\hline Culture positive $^{a}$ & $1(14.3)$ & $10(62.5)$ & .07 \\
\hline Multidrug-resistant bacteria & $1(14.3)$ & 7 (43.8) & .34 \\
\hline Adequate empirical antibiotics & $4(57.1)$ & $14(87.5)$ & .14 \\
\hline Septic shock & $2(28.6)$ & $5(31.3)$ & .38 \\
\hline Infection resolution & $4(57.1)$ & $12(75.0)$ & .63 \\
\hline \multirow{2}{*}{\multicolumn{4}{|c|}{$\begin{array}{l}\text { New episodes of ACLF, } \mathrm{n}(\%) \\
\text { Patients with ACLF, } \mathrm{n}(\%)\end{array}$}} \\
\hline & & & \\
\hline ACLF-1 & $6(9.8)$ & $6(10.5)$ & .86 \\
\hline ACLF-2 & $5(8.2)$ & $5(8.8)$ & \\
\hline ACLF-3 & $1(1.6)$ & $2(3.5)$ & \\
\hline Overall ACLF & $12(19.7)$ & $13(22.8)$ & .68 \\
\hline Potential mechanisms of the new ACLF & & & .17 \\
\hline Recurrence of a resolved ACLF & $2(3.3)$ & 0 & \\
\hline Precipitated by baseline bacterial infection ${ }^{b}$ & $5(8.2)$ & $5(8.8)$ & \\
\hline Precipitated by new bacterial infection & $1(1.6)$ & $4(7.0)$ & \\
\hline Unknown mechanism & $4(6.6)$ & $4(7.0)$ & \\
\hline \multicolumn{4}{|l|}{ Course of ACLF } \\
\hline Resolution & $3(4.9)$ & $6(10.5)$ & .39 \\
\hline \multicolumn{4}{|l|}{ Other clinical events, n (\%) } \\
\hline Variceal bleeding & $4(6.6)$ & $2(3.5)$ & .45 \\
\hline Nonvariceal bleeding & $4(6.3)$ & $3(5.3)$ & .77 \\
\hline Atrial fibrillation & $2(3.3)$ & 0 & .50 \\
\hline Pulmonary edema & $4(6.6)$ & $2(3.5)$ & .45 \\
\hline \multicolumn{4}{|l|}{ Liver transplantation and mortality, $\mathrm{n}(\%)$} \\
\hline Liver transplantation during hospitalization & $1(1.6)$ & $4(7.0)$ & .20 \\
\hline Liver transplantation by 90 days & $2(3.3)$ & $6(10.5)$ & .15 \\
\hline Hospital mortality & $8(13.1)$ & $6(10.5)$ & .66 \\
\hline 90-day mortality ${ }^{C}$ & $17(27.9)$ & $13(22.8)$ & .41 \\
\hline
\end{tabular}

NOTE. Analyses were performed on an intention-to-treat basis.

ACLF, acute-on-chronic liver failure.

${ }^{a}$ In the albumin-plus-antibiotics group there was 1 infection caused by extended-spectrum $\beta$-lactamase Escherichia coli. In the antibiotics-alone group, there were 3 infections caused by vancomycin-susceptible Enterococcus faecium, 3 by E coli ( 1 extended-spectrum $\beta$-lactamase), 2 Klebsiella pneumoniae ( 1 extendedspectrum $\beta$-lactamase, 1 carbapenem-resistant), and 1 by vancomycin-resistant $E$ faecium.

${ }^{b}$ In these cases, ACLF developed during the next 7 days after inclusion with no new infections or other clinical events within this period.

${ }^{c}$ Seven patients were lost to follow-up at 90 days (5 in the albumin-plus-antibiotics group and 2 in the antibiotics-alone group).

Another patient received only $20 \mathrm{~g}$ of albumin on Days 1 and 3. Additional data on albumin treatment are shown in the Supplementary Material.

\section{Primary Outcome}

Death in hospital occurred in 8 patients $(13.1 \%)$ in the study group, and 6 patients $(10.5 \%)$ in the control group (hazard ratio in the study group, 1.2; 95\% confidence interval, $0.5-3.4 ; P=.7789$ ).

\section{Secondary Outcomes}

Effects of treatment on changes in baseline data. Albumin concentration increased significantly only in the study group (Table 2). However, it reached normal levels in 
only 12 out of the 49 patients with hypoalbuminemia at enrollment (Figure 1A). Patients of the study group significantly improved kidney and circulatory function within the first 7 days after enrollment (Table 2, Figure $1 B$ ). These patients also developed a significant suppression of systemic inflammation (decrease in WBC [Figure 1C], CRP, and plasma IL6 concentrations). In contrast, patients from the control group only experienced a significant decrease in CRP. Changes in these inflammatory parameters were, however, not significantly different in the between-group comparison (Table 2).

Most patients received adequate empiric antibiotic therapy (Table 2). Resolution of the baseline infection was obtained in 107 patients (90.7\%), with similar rates between groups. Resolution of infection could not be determined in 3 patients ( 2 in the study group) who were discharged under antibiotic therapy and were lost to follow-up. The remaining 8 patients (6.8\%) suffering from pneumonia $(n=4)$, bacteremia $(n=2)$, and cellulitis and UTI ( $\mathrm{n}=1$, each) died before resolution of the infection. Six patients in the study group and 2 in the control group developed septic shock.

Most patients with ACLF at enrollment (17 out 26; $65.4 \%$ ) resolved the syndrome. Resolution of ACLF was significantly higher in the study group $(82.4 \%$ vs $33.3 \%$; $P=.03$; Figure $1 D$ ). Resolution of KD at enrollment was similar in both groups (70\% and 60\%, respectively). Overall, the percentage of patients who resolved ACLF or $\mathrm{KD}$ in the 2 groups was $77.8 \%$ and $42.9 \%$, respectively $(P=.03)$.

Effects of treatment on the development of new events during hospitalization and on 90-day survival. The median (interquartile range) duration of hospitalization was $11(6-27)$ and 14 (8-27) days in the study and control groups $(P=\mathrm{NS})$, respectively. Twenty patients developed 23 episodes of nosocomial bacterial infections (Table 3). The most frequent infections were pneumonia and bacteremia ( $\mathrm{n}=5$ each). The rate of newly developed bacterial infections (proven and unproven) was significantly lower in the study group $(9.8 \%$ vs $24.6 \%$; $P=.03$; Table 3 ). The rate of newly developed proven bacterial infections was also significantly lower $(P=$ $.007)$ in the study group $(6.6 \%$, vs $24.6 \%)$. After considering liver transplantation as a competing event, the cumulative incidence of new bacterial infection was significantly lower in the study group with a subdistribution hazard ratio of 0.26 (95\% confidence interval, 0.09-0.77) (Figure 1E).

Twelve patients in the study group and 13 in the control group developed an episode of ACLF during hospitalization. ACLF grades at diagnosis were similar between groups (Table 3). The most frequent cause of ACLF during hospitalization was bacterial infections, either present at enrollment $(n=10)$ or developing during hospitalization $(\mathrm{n}=5$ ) (Table 3).

Other significant events were variceal $(n=6)$ and nonvariceal $(n=7)$ hemorrhage, atrial fibrillation $(n=2)$, and pulmonary edema $(n=6)$. There were no significant between-group differences in the overall frequency of these events $(23 \%$ vs $12 \% ; P=.13)$. Side effects potentially related with volume overload (pulmonary edema, atrial fibrillation, and variceal bleeding) were more frequent in the study group, although difference was not statistically significant ( $16 \%$ vs $7 \% ; P=.12)$.

One patient in the study group and 4 in the control group were transplanted during hospitalization, and 1 and 2 additional patients, respectively, were transplanted within the first 90-day period after enrollment. Ninety-day mortality rate was $27.9 \%$ in the study and $22.8 \%$ in the control group $(P=.41)$. The cumulative incidence of death at 90-days did not differ between the 2 study groups, with a subdistribution hazard ratio of $1.22(95 \%$ confidence interval, $0.60-2.50)$ (Figure $1 F$ ).

\section{Results of the Per-Protocol Analysis}

Eleven patients, 5 in the study group and 6 in the control group, presented relevant protocol deviations and were excluded from the per-protocol analysis. Results in the per-protocol population were similar to those observed in the ITT population (Supplementary Tables 5-7, Supplementary Figure 2).

\section{Risk Factors for Relevant Events During Hospitalization, Including Death}

Factors obtained at enrollment showing significant association with relevant events occurring during hospitalization, in-hospital, and 90-day mortality are indicated in Supplementary Table 8.

\section{Discussion}

The 2 RCTs so far published assessing albumin treatment in patients with non-SBP infections ${ }^{7,8}$ were important in the design of our trial. First, we chose hospital mortality instead of 90-day mortality rate as the main endpoint because most patients in these trials died within the first 30 days. Second, because mortality rate in these studies was low, ${ }^{7,8}$ we selected patients with a potential high hospital mortality rate. ${ }^{9}$

The current study did not show significant differences in in-hospital and 90-day mortality rates between treatment arms, confirming the results of the prior RCTs. However, our trial disclosed outstanding findings that have never been reported.

A most relevant feature, which affects the interpretation of the main clinical results of the trial, was that the combined prevalence at enrollment of 2 important predictors of mortality in patients with decompensated cirrhosis (ACLF and KD) was significantly higher in the study group. However, ACLF at enrollment was the most accurate predictor of hospital and 90-day mortality in the trial. Therefore, disease severity at enrollment was 
significantly greater in those patients assigned to the study group. This might explain the lack of effect of albumin treatment on mortality despite inducing significant beneficial effects on important pathophysiological mechanisms and complications.

First, we observed a potential beneficial effect of albumin on systemic inflammation within the first 7 days of treatment. Any decrease in inflammatory biomarkers in our patients could be attributed to a rapid control of the infection. However, whereas treatment with antibiotics alone was associated with a significant decrease in CRP but not in WBC or IL6, treatment with antibiotic plus albumin led to significant reduction in levels of these 3 inflammatory biomarkers. Changes were, nevertheless, not significantly different in the between-group comparison.

Second, treatment with antibiotics plus albumin but not with antibiotics alone was associated with improvement in renal and circulatory function as indicated by a significant increase in mean arterial pressure and serum sodium and decrease in PRC and creatinine in the study group but not in the control group.

Third, treatment with antibiotics plus albumin was associated with a surprisingly high-resolution rate of the ACLF present at enrollment (82.3\%), significantly higher than that observed in the control group (33.3\%). This resolution rate is higher than that observed in the Chronic Liver Failure Acute-on-Chronic Failure in Cirrhosis (CANONIC) study in patients not receiving albumin, ${ }^{1}$ suggesting that albumin treatment, if administered early after diagnosis, may increase the resolution of ACLF.

Fourth and most important beneficial effect of albumin treatment was that prevalence of proven bacterial infections during hospitalization was 4-fold lower in patients receiving albumin, suggesting a preventive effect of albumin on nosocomial bacterial infections. In contrast, albumin treatment did not prevent ACLF development during hospitalization.

The beneficial effects of albumin treatment observed in the study could be related to its dampening action on excessive systemic proinflammatory signals. Excessive proinflammatory response is compensated with the activation of anti-inflammatory mediators to prevent immunopathology, favoring secondary infections. ${ }^{5,12} \mathrm{Al}$ bumin could modulate this homeostatic compensatory reaction and therefore protect from the development of nosocomial infections.

Our trial has several limitations. The statistical power to detect a potential reduction in hospital mortality in patients treated with albumin was low because only 118 patients could be enrolled into the study. Differences in the exclusion criteria between our trial and the cohorts included in our pilot investigation ${ }^{13-15}$ could have contributed to overestimation of the recruitment rate and hospital mortality. Finally, we did not obtain good matching between groups. Inclusion of less sick patients in the control group could explain the lower than expected hospital mortality rate in this group $(10.5 \%)$. However, the strength of the study was the study design and inclusion of clinically important endpoints, including the clinical effects of albumin treatment on patients' clinical course during hospitalization.

As expected, albumin treatment significantly increased serum albumin concentration but only a minority of patients with hypoalbuminemia normalized albumin concentration. The inhibition of the hepatic synthesis of albumin by bacterial infection may account for this feature. In addition, the half-life of circulating albumin is markedly reduced when, as it occurs in cirrhosis, there is impairment in the molecular structure of the protein. ${ }^{16}$ We have observed that the immunomodulatory effect of albumin is highly dependent on the post-treatment serum albumin concentration. ${ }^{17}$ Therefore, current albumin dosage in patients with cirrhosis with bacterial infections may be too simplistic. A more rational approach may consist of the administration of a priming dose followed by additional periodical doses tailored by the serum albumin concentration.

Finally, the frequency of pulmonary edema was slightly higher in patients receiving albumin. When all events potentially related to volume overload were considered together, there was a trend toward a higher rate of these side effects in the albumin group.

In summary, in this trial involving patients with advanced cirrhosis and infections unrelated to SBP, inhospital mortality was not different among patients who received albumin plus antibiotics and those who received antibiotics only. This occurred despite the beneficial effects of albumin treatment on circulatory and renal dysfunction, resolution of baseline ACLF, and prevention of nosocomial infections. Disease severity at inclusion, however, was greater in the group of patients treated with albumin. Further appropriately designed and powered RCTs are needed to ascertain if albumin treatment decreases mortality in patients with cirrhosis with non-SBP bacterial infections.

\section{Supplementary Material}

Note: To access the supplementary material accompanying this article, visit the online version of Clinical Gastroenterology and Hepatology at www.cghjournal.org, and at https://doi.org/10.1016/j.cgh.2019.07.055.

\section{References}

1. Moreau R, Jalan R, Gines $P$, et al. Acute-on-chronic liver failure is a distinct syndrome that develops in patients with acute decompensation of cirrhosis. Gastroenterology 2013; 144:1426-1437.

2. Clària J, Stauber RE, Coenraad MJ, et al. Systemic inflammation in decompensated cirrhosis: characterization and role in acuteon-chronic liver failure. Hepatology 2016;64:1249-1264.

3. Arroyo V, Moreau R, Kamath PS, et al. Acute-on-chronic liver failure in cirrhosis. Nat Rev Dis Primers 2016.
1103

1104

1105

1106

1107

1108

1109

1110

1111

1112

1113

1114

1115

1116

1117

1118

1119

1120

1121

1122

1123

1124

1125

1126

1127

1128

1129

1130

1131

1132

1133

1134

1135

1136

1137

1138

1139

1140

1141

1142

1143

1144

1145

1146

1147

1148

1149

1150

1151

1152

1153

1154

1155

1156

1157

1158

1159

1160 
4. Bernardi M, Moreau R, Angeli P, et al. Mechanisms of decompensation and organ failure in cirrhosis: from peripheral arterial vasodilation to systemic inflammation hypothesis. J Hepatol 2015;63:1272-1284.

5. Fernández $\mathrm{J}$, Acevedo $\mathrm{J}$, Wiest $\mathrm{R}$, et al. Bacterial and fungal infections in acute-on-chronic liver failure: prevalence, characteristics and impact on prognosis. Gut 2018;67:1870-1880.

6. Sort P, Navasa M, Arroyo V, et al. Effect of intravenous albumin on renal impairment and mortality in patients with cirrhosis and spontaneous bacterial peritonitis. N Engl J Med 1999; 341:403-409.

7. Guevara M, Terra C, Nazar A, et al. Albumin for bacterial infections other than spontaneous bacterial peritonitis in cirrhosis. A randomized, controlled study. J Hepatol 2012;57:759-765.

8. Thevenot $\mathrm{T}$, Bureau $\mathrm{C}$, Oberti $F$, et al. Effect of albumin in cirrhotic patients with infection other than spontaneous bacterial peritonitis. J Hepatol 2015;62:822-830.

9. Fernández J, Acevedo J, Prado V, et al. Clinical course and short-term mortality of cirrhotic patients with infections other than spontaneous bacterial peritonitis. Liver Int 2017; 37:385-395.

10. Fernández J, Navasa M, Planas R, et al. Primary prophylaxis of spontaneous bacterial peritonitis delays hepatorenal syndrome and improves survival in cirrhosis. Gastroenterology 2007; 133:818-824.

11. Jalan R, Saliba F, Pavesi M, et al. Development and validation of a prognostic score to predict mortality in patients with acute-onchronic liver failure. J Hepatol 2014;61:1038-1047.

12. Delano MJ, Ward PA. The immune system's role in sepsis progression, resolution, and long-term outcome. Immunol Rev 2016;274:330-353.

13. Fernández $\mathrm{J}$, Navasa $\mathrm{M}$, Gómez $\mathrm{J}$, et al. Bacterial infections in cirrhosis: epidemiological changes with invasive procedures and norfloxacin prophylaxis. Hepatology 2002; 35:140-148.

14. Fernández $\mathrm{J}$, Acevedo $\mathrm{J}$, Castro $\mathrm{M}$, et al. Prevalence and risk factors of infections by multiresistant bacteria in cirrhosis: a prospective study. Hepatology 2012;55:1551-1561.

15. Acevedo J, Fernández J, Prado $\mathrm{V}$, et al. Relative adrenal insufficiency in decompensated cirrhosis. Relationship to short-term risk of severe sepsis, hepatorenal syndrome, and death. Hepatology 2013;58:1757-1765.

16. Arroyo V, García-Martínez R, Salvatella X. Human serum albumin, systemic inflammation, and cirrhosis. J Hepatol 2014; 61:396-407.

17. Fernández $\mathrm{J}$, Clària $\mathrm{J}$, Amorós $\mathrm{A}$, et al. Effects of albumin treatment on systemic and portal hemodynamics and systemic inflammation in patients with decompensated cirrhosis. Gastroenterology 2019.

\section{Reprint requests}

Address requests for reprints to: Javier Fernández, MD, PhD, Liver Unit, Hospital Clínic, Villarroel 170,08036, Barcelona, Spain.e-mail:Jfdez@clinic.ub. es; fax: 34-93-4515522.

\section{Conflicts of interest}

This author discloses the following: Javier Fernandez has received research support from Grifols. The other authors disclose no conflicts. The EASL-CLIF Consortium is a network of 101 European University hospitals supported by the European Foundation for the Study of Chronic Liver Failure (EF-Clif). EF-Clif is a private nonprofit organization aimed at improving clinical and translational research in cirrhosis. The scientific agenda of the EASL-CLIF Consortium and the specific research protocols are made exclusively by the Steering Committee members without any participation of pharmaceutical companies.

\section{Funding}

The study was supported by the European Foundation for the Study of Chronic Liver Failure (EF-Clif). EF-Clif received unrestricted donations from Grifols and Cellex Foundations and is partner or contributor in several projects of the EU Q17 Horizon 2020 research program. 


\section{Supplementary Appendix}

\section{Patients and Methods}

Exclusion Criteria. Exclusion criteria were infections evolving for $>72$ hours, septic shock, ${ }^{1}$ endocarditis, fungal infection, severe acute respiratory distress syndrome $\left(\mathrm{PaO}_{2} / \mathrm{FiO}_{2} \leq 100\right)$, active or recent variceal bleeding (unless controlled for $>48$ hours), type- 1 HRS (International Ascites Club criteria), ${ }^{2}$ ACLF grade-3 (3 or more organ failures [OFs] according to the Canonic Study criteria), ${ }^{3}$ renal-replacement therapy, malignancy (except for hepatocellular carcinoma within Milan criteria or nonmelanocytic skin cancer), moderate or severe chronic heart failure (New York Heart Association functional class II, III, or IV), severe chronic pulmonary disease (Global Initiative for Chronic Obstructive Lung Disease IV), previous liver transplantation, severe psychiatric disorders that prevent the patient from making autonomous decisions, human immunodeficiency virus infection (except for patients under antiretroviral therapy with undetectable viral load, CD4 levels $>200 / \mathrm{mm}^{3}$, and no previous history of opportunistic infections), contraindications to albumin (allergy, signs of pulmonary edema), albumin administration $(\geq 80 \mathrm{~g})$ in the last 2 days, SBP coinfection, administration of any investigational drug within 90 days before randomization, premenopausal women not practicing an acceptable method of birth control, refusal to participate, patients who could not provide prior informed consent and when there was documented evidence that the patient had no legal surrogate decision maker and it seemed unlikely that the patient would regain consciousness or sufficient ability to provide delayed informed consent, and physician and team not committed to intensive care if needed (DNR code).

\section{Definitions}

Acute-on-chronic liver failure. The diagnosis of ACLF and its grades were performed according to the Canonic study criteria. ${ }^{3}$ ACLF was defined as the association of acute decompensation (defined as ascites, encephalopathy, variceal hemorrhage, or any combination of these), single or multiple $(\geq 2)$ OFs, and high short-term (28day) mortality risk $(>15 \%)$. OF was defined for each of the 3 major organ systems (liver, kidney, brain, coagulation, circulation, and respiration) as a CLIF-Consortium OF score of 2 or 3 (on a scale ranging from 1 to 3 for each organ system) ${ }^{3}$ (Supplementary Table 1). In brief, for nonkidney organ systems, a score of 1 indicated normal of relatively preserved organ function; a score of 2 , organ dysfunction (OD), including liver dysfunction, brain dysfunction, coagulation dysfunction, circulatory dysfunction, and respiratory dysfunction); and a score of 3 , OF. For the kidney, scores of 2 and 3 indicated kidney failure. Among patients with a kidney score of 1 , those who had serum creatinine levels ranging from 1.5-1.9 $\mathrm{mg} / \mathrm{dL}$ were defined as having KD.

According to Canonic criteria, patients with acute decompensation are classified into 4 groups according to the number and type of OFs 1 (Supplementary Table 2):

1. ACLF-3: 3-6 OFs (mortality risk, 75\%)

2. ACLF-2: 2 OFs (mortality risk, 32\%)

3. ACLF-1: Single kidney failure, or single nonkidney $\mathrm{OF}$ if associated with KD and/or brain dysfunction (mortality risk, 22\%)

4. No ACLF: None of these characteristics (mortality risk, 4.6\%). Please note that patients from this group may have single nonkidney OF (Supplementary Table 1) or single or combined OD.

Resolution of ACLF was defined as a decrease from any grade of ACLF to no ACLF. ${ }^{4}$

Bacterial infections. Definitions and diagnostic criteria of infections were the following. SBP: polymorphonuclear (PMN) cell count in ascitic fluid $\geq 250$ / $\mathrm{mm}^{3}$; UTI: abnormal urinary sediment $(>10$ leukocytes/ field) or a positive reagent strip and positive urinary culture or uncountable leukocytes per field if negative cultures; spontaneous bacteremia: positive blood cultures and no cause of bacteremia; secondary bacteremia: catheter-related infection (positive blood and catheter cultures), and bacteremia occurring within 24 hours after an invasive procedure; pneumonia: clinical signs of infection and new infiltrates on chest radiograph; bronchitis: clinical features of infection, no radiographic infiltrates, and positive sputum culture; cellulitis: clinical signs of infection associated with swelling, erythema, heat, and tenderness in the skin; cholangitis: cholestasis, right upper quadrant pain and/or jaundice, and radiologic data of biliary obstruction; spontaneous bacterial empyema: PMN count in pleural fluid $\geq 500 / \mathrm{mm}^{3}(250$ / $\mathrm{mm}^{3}$ if positive culture); secondary peritonitis: PMN count in ascitic fluid $\geq 250 / \mathrm{mm}^{3}$ and evidence (abdominal computed tomography/surgery) of an intraabdominal source of infection; Clostridium difficile infection: positive stool toxin in a patient with diarrhea; unproved bacterial infection: presence of fever $\left(\geq 38^{\circ} \mathrm{C}\right)$ and leukocytosis (white blood cell count $\geq 12.000 / \mathrm{mm}^{3}$ ) requiring antibiotic therapy without any identifiable source. Infections diagnosed at admission or within 2 days after admission were classified as health care-associated (HCA) in patients with a prior contact with the health care environment (hospitalization or short-term admission for at least 2 days in the previous 90 days, residence in a nursing home or a long-term care facility, or chronic hemodialysis). The remaining infections were considered community-acquired (CA) when they were present at admission or developed within the first 48 hours after hospitalization and nosocomial when the diagnosis was made thereafter. ${ }^{5}$ 
MDR was defined as acquired nonsusceptibility to at least 1 agent in 3 or more antimicrobial categories. ${ }^{6}$ The following bacteria were considered MDR in the current study: extended-spectrum $\beta$-lactamase (mainly Escherichia coli and Klebsiella pneumoniae) or desrepressed chromosomic AmpC ß-lactamase-producing Enterobacteriaceae (Enterobacter, Citrobacter, and Proteus spp), carbapenem-resistant $K$ pneumoniae, carbapenemresistant Pseudomonas aeruginosa, Stenotrophomonas maltophilia, carbapenem-resistant Acinetobacter baumanii, methicillin-resistant Staphylococcus aureus (MRSA), and vancomycin-susceptible Enterococcus faecium.

Patients were considered to have systemic inflammatory response syndrome (sepsis) if they fulfilled at least 2 of the following criteria: (1) core temperature $>38^{\circ} \mathrm{C}$ or $<36^{\circ} \mathrm{C}$; (2) heart rate $>90$ beats/minute; (3) respiratory rate $>20$ breaths/minute in the absence of hepatic encephalopathy; and (4) white blood cell count $>12.000$ or $<4000 / \mathrm{mm}^{3}$, or differential count showing $\geq 10 \%$ immature PMN neutrophils. Septic shock was diagnosed by the presence of data compatible with systemic inflammatory response syndrome and need of vasopressor drugs in the setting of hypotension. ${ }^{1}$ Recently defined sepsis criteria were not applied in the current study because they were proposed after the end of the Canonic Study.

Infections were considered cured when all clinical signs of infection disappeared and on the presence of: (1) urinary infections: normal urine sediment and negative urine culture; (2) spontaneous or secondary bacteremia: negative control cultures after antibiotic treatment; (3) pneumonia: normal chest radiograph and negative control cultures if positive at diagnosis; (4) bronchitis: negative bronchial aspirate/sputum culture; (5) cellulitis: normal physical examination of the skin and negative control cultures if positive at diagnosis; (6) cholangitis: improvement of cholestasis, resolution of clinical symptoms, and negative control cultures if positive at diagnosis; and (7) SBP and spontaneous bacterial empyema: PMN cell count in ascitic/pleural fluid $<250 /$ $\mathrm{mm}^{3}$ and negative control cultures if positive at diagnosis. Resolution of the rest of infections was based on conventional clinical criteria. ${ }^{5}$

The criteria used to consider an initial antibiotic therapy appropriate were the following: culture-positive infections, if an antibiotic with an in vitro activity appropriate for the isolated pathogen or pathogens was administered at diagnosis of infection; and culturenegative infections, when the antibiotic strategies administered at the time of infection diagnosis solved the infection without need for further escalation. Otherwise, the initial therapy was considered inappropriate. ${ }^{5}$

\section{Empirical Antibiotic Treatment Suggested in the Study}

Empirical antibiotic treatment should be administered within the first 6 hours after infection diagnosis and will be different for infections acquired in the community (CA infections) and HCA or nosocomial infection because of the higher prevalence of multiresistant bacteria in the later infections. Treatment schedule will be adapted to the local epidemiologic pattern of multiresistance. The suggested empirical treatment in this protocol as follows.

Community-acquired infections. CA infections will be treated as follows: ceftriaxone for urinary and suspected bacterial infection; ceftriaxone plus cloxacillin or amoxicillin clavulanic-acid for cellulitis; piperacillintazobactam for cholangitis; and ceftriaxone and a macrolide or levofloxacin in patients with pneumonia. Ceftriaxone plus clindamycin will be administered in case of aspiration pneumonia.

Health care-associated and nosocomial infections. Empirical antibiotic regimen in HCA or nosocomial infections will be the following: carbapenem for urinary and suspected bacterial infection (plus a glycopeptide in areas with a high prevalence of $E$ faecium); imipenem for cholangitis (plus a glycopeptide in areas with a high prevalence of $E$ faecium); antipseudomonic carbapenem (meropenem, imipenem, or doripenem) or ceftazidime plus glycopeptide for cellulitis; and antipseudomonic carbapenem or ceftazidime plus ciprofloxacin in patients with pneumonia. Vancomycin, teicoplanin, or linezolid will be added in patients with pneumonia and risk factors for MRSA (ventilator-associated pneumonia, previous antibiotic therapy, nasal MRSA carriage).

Antibiotics will be administered intravenously at standard doses: ceftriaxone, $2 \mathrm{~g}$ at diagnosis followed by $1 \mathrm{~g} / 12-24$ hours; amoxicillin clavulanic-acid, 2-0.4 g/8 hours; cloxacillin, $2 \mathrm{~g} / 6$ hours; clarithromycin, $500 \mathrm{mg} /$ 12 hours; levofloxacin, $500 \mathrm{mg} / 12-24$ hours; clindamycin, $600 \mathrm{mg} / 8$ hours; piperacillin-tazobactam, $4 \mathrm{~g} / 6-8$ hours; meropenem, $1 \mathrm{~g} / 8$ hours; doripenem, $0.5 \mathrm{~g} / 8$ hours; imipenem, 0.5-1 g/6-8 hours; ertapenem, $1 \mathrm{~g} /$ 12-24 hours; ceftazidime, 2 g/8 hours; ciprofloxacin, 400 mg/8-12 hours; vancomycin, 15-20 mg/kg/8-12 hours; teicoplanin, $6 \mathrm{mg} / \mathrm{kg}$; and linezolid, $600 \mathrm{mg} / 12$ hours. Doses will be adjusted to renal function (glomerular filtration rate will be estimated by the Cockcroft-Gault or the MDRD equations following local guidelines). De- Q11 escalation to the most appropriate antibiotic should be done early after knowing the results of the microbiologic tests.

Antibiotic treatment will be maintained until the disappearance of signs and symptoms of infection, normalization of the white blood cell count, and negative cultures. Patients with positive cultures at infection diagnosis will have to have control negative cultures before stopping antibiotics. Suggested duration of treatment in CA infections will be of 7 days except for pyelonephritis, cholangitis, and cellulitis (10 days). Suggested duration of treatment in HCA and nosocomial infections will be of 10 days, except for pneumonia and cellulitis caused by multiresistant bacteria (14 days). 


\section{Assessment of Disease Severity at Enrollment}

To avoid the limitations of the previous trials regarding patients' severity, only cases with acute decompensation and significant liver and renal impairment, as prespecified by high levels of serum bilirubin and/or creatinine and/or low plasma sodium levels, were enrolled in our trial. Patient severity at enrollment was initially planned to be assessed by these measurements and by the Model for End-Stage Liver Disease and Child-Pugh scores. However, investigations published between the study design and data analysis ${ }^{7-10}$ showed important limitations of these measurements and scores. Moreover, the diagnostic criteria of ACLF proposed by the Canonic study leave many patients with single nonkidney OF or with single or combined OD in the nonACLF group (Supplementary Tables 1 and 2). Recent data indicate that these patients are at higher risk of developing worse clinical course or dying than those without $\mathrm{OF}$ or OD. ${ }^{11}$ However, KD was found to be an accurate marker of high short-term mortality $(19.2 \%$ vs $5.7 \%$ in patients with and without $\mathrm{KD}$, respectively; $P=$ .02 ) in a pilot investigation in 496 patients without ACLF from Canonic study database (Supplementary Table 3). Based on these results, we decided to add ACLF and KD at enrollment as indicators of disease severity before data analysis.

\section{Results}

\section{Microbiology of Bacterial Infections Diagnosed at Inclusion}

$E$ coli was the most frequently isolated organism (34.8\%), followed by $S$ aureus (14.5\%); K pneumoniae (10.1\%); and Streptococcus viridans, Enterobacter spp, and Enterococcus faecalis (4.3\% each). Twenty-six of the 69 organisms isolated in the study $(37.7 \%)$ were multidrug-resistant organisms. They were isolated in 24 infections $20.3 \%$ of all infections, $38.1 \%$ of culturepositive infections). As a whole, extended-spectrum $\beta$ lactamase-producing $E$ coli was the most frequent multidrug-resistant organism reported $(n=9)$, followed by MRSA $(\mathrm{n}=4)$ (Supplementary Table 4$)$.

\section{Albumin Administration}

Albumin treatment was initiated after the first 8 hours following randomization in 4 patients. Overall, the median albumin doses (interquartile range [IQR]) administered on Day 1 and Day 3 were 100 (90-110 g) and 62.5 (60-80 g), respectively. The median difference (IQR) between the scheduled dose and the administered dose of albumin on Day 1 was $0(-2.5$ to $0.5 \mathrm{~g})$ and $0(0-1$ g) on Day 3. Nine patients (6 in the control group and 3 in the study group) received relevant albumin doses $(\geq 1$ $\mathrm{g} / \mathrm{kg}$ body weight) within the first week after randomization for the treatment of complications other than the initial infection. The median albumin doses (IQR) administered were 80 (70-100 g) in the study group and $60 \quad(55-90 \mathrm{~g})$ in the control group, respectively.

\section{Risk Factors for Relevant Events During Hospitalization, Including Death}

Factors obtained at enrollment showing significant association with relevant events occurring during hospitalization, in-hospital, and 90-day mortality are indicated in Supplementary Table 8. Albumin treatment was independently associated with lower prevalence of proven bacterial infections. Diabetes was independently related to the development of pulmonary edema. Hepatic encephalopathy and severity of systemic inflammation (as estimated by WBC) at enrollment were independently associated with a higher prevalence of ACLF. Finally, ACLF at enrollment was the only independent predictor of in-hospital mortality, the primary outcome of the trial, and was also identified as an independent predictor of 90-day mortality together with bacteremia and prothrombin index.

Baseline serum albumin levels were lower in patients included in the study group who developed ACLF or bacterial infections during hospitalization, although differences were not statistically significant (Supplementary Table 9).

Supplementary Table 10 shows details concerning the main events occurring during hospitalization in patients who died and their causes of death. Death was related with lack of resolution of bacterial infections in 11 patients (8 corresponding to infections detected at enrollment).

\section{References}

1. American College of Chest Physicians/Society of Critical Care Medicine Consensus Conference. Definitions for sepsis and organ failure and guidelines for the use of innovative therapies in sepsis. Crit Care Med 1992;20:864-874.

2. Angeli $P$, Gines $P$, Wong $F$, et al. Diagnosis and management of acute kidney injury in patients with cirrhosis: revised consensus recommendations of the International Club of Ascites. Gut 2015; 64:531-537.

3. Moreau R, Jalan R, Gines P, et al. Acute-on-chronic liver failure is a distinct syndrome that develops in patients with acute decompensation of cirrhosis. Gastroenterology 2013; 144:1426-1437.

4. Gustot T, Fernández J, Garcia E, et al. Clinical course of acuteon-chronic liver failure syndrome and effects on prognosis. Hepatology 2015;62:243-252.

5. Fernández J, Acevedo J, Wiest R, et al. Bacterial and fungal infections in acute-on-chronic liver failure: prevalence, characteristics and impact on prognosis. Gut 2018;67:1870-1880.

6. Magiorakos A-P, Srinivasan A, Carey RB, et al. Multidrugresistant, extensively drug-resistant and pandrug-resistant bacteria: an international expert proposal for interim standard 


\section{Supplementary}

Figure 1. Flow chart of the study. Overall, 534 patients were assessed for eligibility, and 433 subjects were excluded. A total of 136 patients were enrolled. Eighteen patients were considered inclusion errors. The final ITT population consisted of 118 patients, 61 in the study group and 57 in the control group. Eleven patients, 5 in the study group and 6 in the control group, presented relevant protocol deviations. Seven patients were lost to follow-up at 90 days. ARDS, acute respiratory distress syndrome; GOLD, Global Initiative for Chronic Obstructive Lung Disease; HCC, hepatocellular carcinoma; HIV, human immunodeficiency virus; NYHA, New York Heart Association; SIRS, systemic inflammatory response syndrome.
534 patients screened until

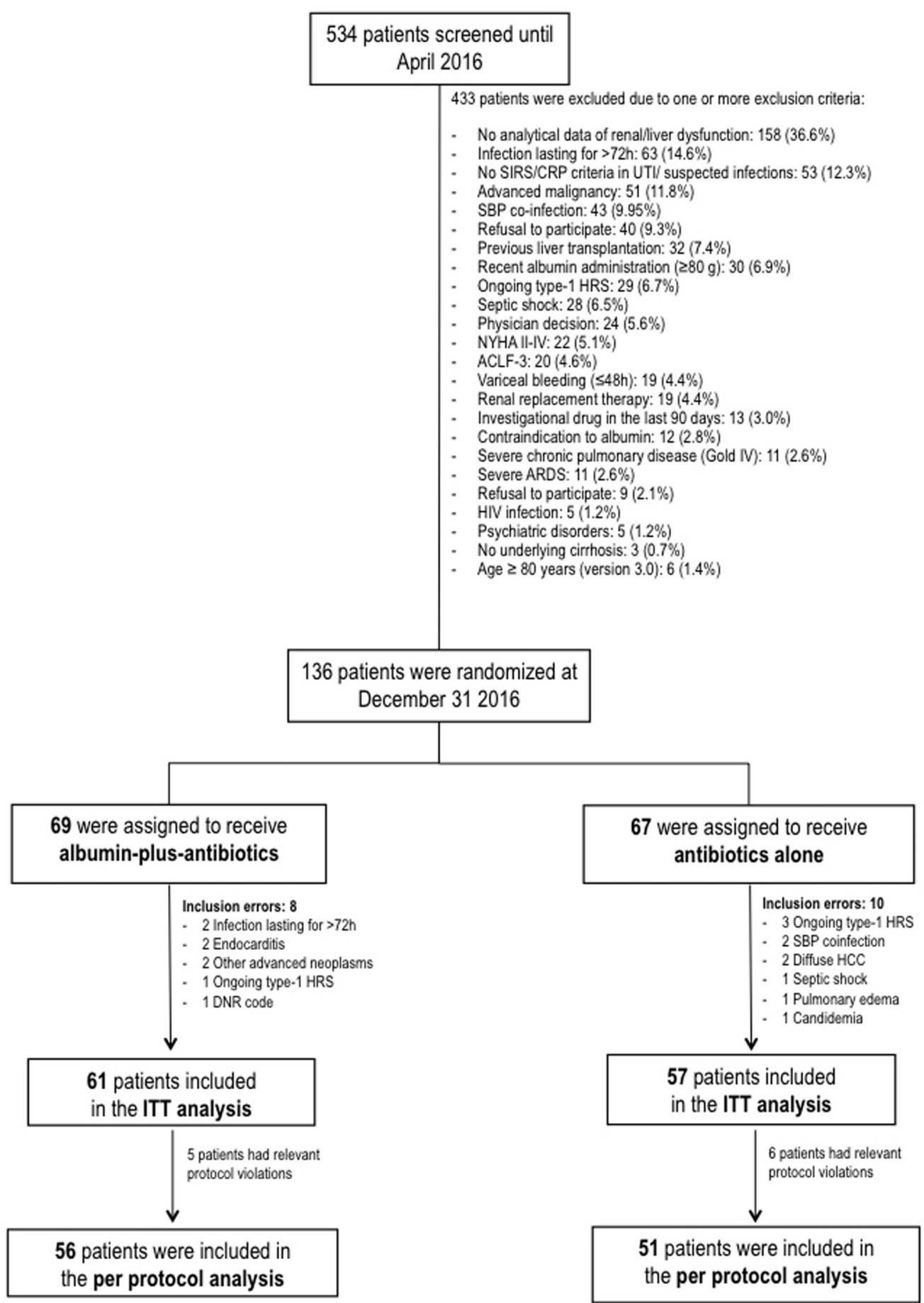

5 patients were lost to follow-up at $90 \cdot d$ April 2016

1683

1684

1685

1686

1687

1688

1689

1690

1691

1692

1693

1694

1695

1696

1697

1698

1699

1700

1701

1702

1703

1704

1705

1706

1707

1708

1709

1710

1711

1712

1713

1714

1715

1716

1717 before and after liver transplantation. Gastroenterology 2018.

11. Trebicka J, Amoros A, Pitarch C, et al. Addressing profiles of systemic inflammation across the different clinical phenotypes of acutely decompensated cirrhosis. Front Immunol (in press). cirrhosis admitted to intensive care unit. Comparison with current models and CLIF-SOFA score. Am J Gastroenterol 2014; 109:554-562. 
ARTICLE IN PRESS

11.e5 Fernández et al

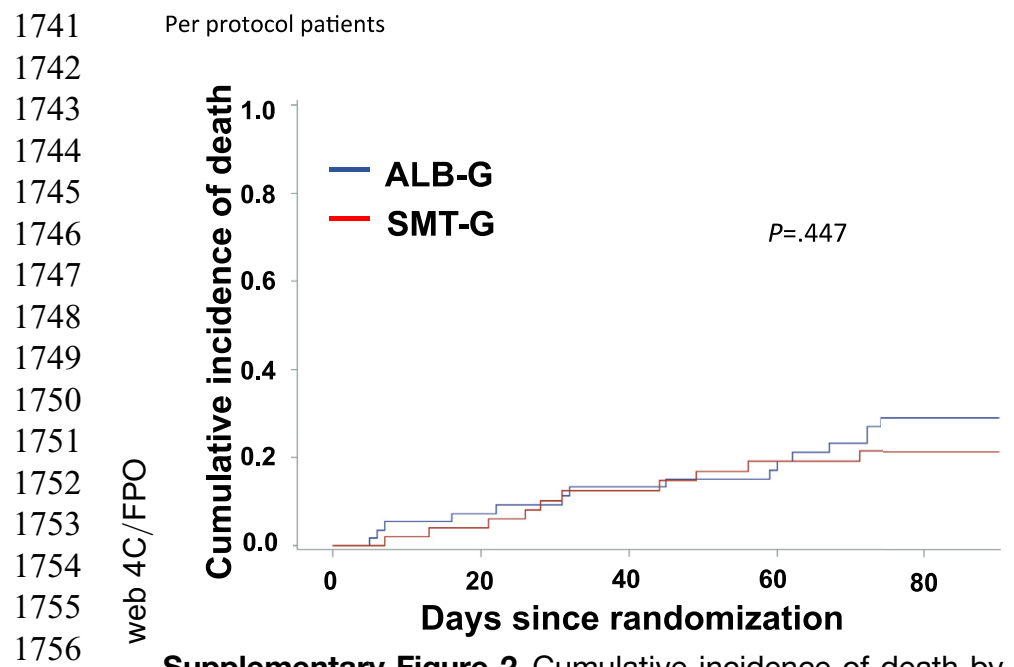

Supplementary Figure 2. Cumulative incidence of death by 90 days in the per-protocol population, when liver transplantation was taken into account as a competing risk of death. At 90 days, the estimated cumulative incidence of death was $28.9 \%$ (95\% confidence interval, $17.2 \%-41.7 \%$ ) in the albumin-plus-antibiotics group and $21.3 \%$ (95\% confidense interval, $10.9 \%-34.1 \%$ ) in the antibiotics-alone group. No significant differences were observed between groups.

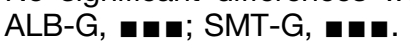

Clinical Gastroenterology and Hepatology Vol. $\square$, No.
1757

1758

1759

1760

1761

1762

1763 Q14

1764

1765

1766

1767

1768

1769

1770

1771

1772

1773

1774

1775

1776

1777

1778

1779

1780

1781

1782

1783

1784

1785

1786

1787

1788

1789

1790

1791

1792

1793

1794

1795

1796

1797

1798
1799

1801

1802

1803

1804

1805

1806

1807

1808

1809

1810

1811

1812

1813

1814

1815

1816

1817

1818

1819

1820

1821

1822

1823

1824

1825

1826

1827

1828

1829

1830

1831

1832

1833

1834

1835

1836

1837

1838

1839

1840

1841

1842

1843

1844

1845

1846

1847

1848

1849

1850

1851

1852

1853

1854

1855

1856

FLA 5.6.0 DTD $\square$ YJCGH56661 proof $\square 27$ September $2019 \square$ 12:10 am $\square$ ce CJ 
Supplementary Table 1. Diagnostic Criteria of Organ Failures and Organ Dysfunctions According to the CLIF-Consortium Organ Failure Scoring System

\begin{tabular}{llll}
\hline & \multicolumn{3}{c}{ Scale assessing the deterioration in organ system functions } \\
\cline { 2 - 4 } \multicolumn{1}{c}{ Organ system } & \multicolumn{1}{c}{1 point } & \multicolumn{1}{c}{2 points } & \multicolumn{1}{c}{3 points } \\
\hline Liver & Bilirubin $<6 \mathrm{mg} / \mathrm{dL}$ & Bilirubin $\geq 6 \mathrm{mg} / \mathrm{dL}$ and $\leq 12 \mathrm{mg} / \mathrm{dL}$ & Bilirubin $>12 \mathrm{mg} / \mathrm{dL}$ \\
Kidney & Creatinine $<2 \mathrm{mg} / \mathrm{dL}$ & Creatinine $\geq 2 \mathrm{mg} / \mathrm{dL}$ and $<3.5 \mathrm{mg} / \mathrm{dL}$ & Creatinine $\geq 3.5 \mathrm{mg} / \mathrm{dL}$ or RRT \\
& Creatinine from 1.5 to $1.9 \mathrm{mg} / \mathrm{dL}$ & & \\
Brain (West-Haven) & Grade 0 & Grade $1-2$ & Grade $3-4$ \\
Coagulation & INR $<2.0$ & $2.0 \leq \mathrm{INR}<2.5$ & INR $\geq 2.5$ \\
Circulation & $\mathrm{MAP} \geq 70 \mathrm{~mm} \mathrm{Hg}$ & $\mathrm{MAP}<70$ & Vasopressor requirement \\
Respiratory $^{2}$ & $>300$ & $>200$ and $\leq 300$ & $\leq 200$ \\
PaO $_{2} / \mathrm{FIO}_{2}$ or $\mathrm{SpO}_{2} / \mathrm{FlO}_{2}$ & $>357$ & $>214$ and $\leq 357$ & $\leq 214$ \\
& & & \\
\hline
\end{tabular}

NOTE. Adapted from Reference 3. Each organ system (liver, kidney, coagulation, brain, circulation, respiration) receives a score ranging from 1 point (normal or close to normal) to 3 points (most abnormal). Highlighted areas in blue reflect the definition of each organ failure. Highlighted areas in orange reflect the definition of each organ dysfunction. The sum of the 6 individual organ scores gives rise to an aggregated score called CLIF-Consortium organ failure score, ranging from 5 to 15 , higher scores indicating more severe disease.

CLIF, chronic liver failure; $\mathrm{FIO}_{2}$, fraction of inspired oxygen; INR, international normalized ratio; MAP, mean arterial pressure; RRT, renal-replacement therapy; $\mathrm{SpO}_{2}$, pulse oximetric saturation.

${ }^{a}$ Patients treated with mechanical ventilation received a respiratory organ failure score of 3 points.

Supplementary Table 2. Mortality Rates by 28 Days According to the Number and Types of In-Patients With or Without Kidney and/or Brain Dysfunction (Canonic Study)

\begin{tabular}{lcc}
\hline & $\begin{array}{c}\text { No kidney dysfunction and } \\
\text { no brain dysfunction }\end{array}$ & $\begin{array}{c}\text { Kidney dysfunction or brain } \\
\text { dysfunction, or both }\end{array}$ \\
\cline { 2 - 3 } Number and types of OF & & Mortality rate (\%) \\
\hline OF absent & 3.6 & 6.2 \\
Single liver failure & 5.9 & 30.3 \\
Single cerebral failure & 8.0 & 20.0 \\
Single coagulation failure & 5.3 & 22.2 \\
Single circulation or single & 6.7 & 28.6 \\
$\quad$ respiratory failure & 15.8 & 24.1 \\
Single kidney failure & 28.8 & 38.7 \\
Two OFs & 86.2 & 61.5 \\
Three OFs or more & &
\end{tabular}

NOTE. Adapted from Reference 1 (main manuscript). The highlighted colored areas show the subgroups of patients with ACLF: in blue ACLF-1, in orange ACLF-2, in yellow ACLF-3.

ACLF, acute-on-chronic liver failure; OF, organ failure. 


\section{1.e7 Fernández et al}

Clinical Gastroenterology and Hepatology Vol. a, No.

Supplementary Table 3. Development of ACLF and Death by 28 Days Among the 496 Patients Without ACLF but With Different Types of Single Organ System Failure or Dysfunction at Enrollment (Canonic Study Patients)

\begin{tabular}{|c|c|c|c|c|}
\hline Organ system & $\begin{array}{l}\text { ACLF development } \\
\text { by } 28 \mathrm{~d}\end{array}$ & $P$ value & 28-d mortality & $P$ value \\
\hline \multicolumn{5}{|l|}{ Liver, n/N (\%) } \\
\hline Liver dysfunction or liver failure only & 12/54 (22.2) & .003 & 4/52 (7.7) & .076 \\
\hline No liver dysfunction or liver failure ${ }^{a}$ & 50/442 (11.3) & & $27 / 427(6.3)$ & \\
\hline \multicolumn{5}{|l|}{ Kidney, n/N (\%) } \\
\hline Kidney dysfunction only & 12/28 (42.9) & $<.00001$ & $5 / 26(19.2)$ & .002 \\
\hline No kidney dysfunction ${ }^{2}$ & 50/468 (10.7) & & 26/453 (5.7) & \\
\hline \multicolumn{5}{|l|}{ Brain, n/N (\%) } \\
\hline Brain dysfunction or failure only & $6 / 85(7.1)$ & .01 & 3/83 (3.6) & .033 \\
\hline No brain dysfunction or brain failure ${ }^{a}$ & 56/411 (13.6) & & 28/396 (7.1) & \\
\hline \multicolumn{5}{|l|}{ Coagulation, n/N (\%) } \\
\hline Coagulation dysfunction or failure only & $1 / 11(9.1)$ & 1.0 & 2/11 (18.2) & .015 \\
\hline No coagulation dysfunction or coagulation failure ${ }^{a}$ & 61/485 (12.6) & & $29 / 468(6.2)$ & \\
\hline
\end{tabular}

NOTE. Among the 496 patients, 62 developed ACLF and 31 died by 28 days. ACLF, acute-on-chronic liver failure.

ancludes patients with or without other organ dysfunction.
2031

2032

2033

2034

2035

2036

2037

2038

2039

2040

2041

2042

2043

2044

2045

2046

2047

2048

2049

2050

2051

2052

2053

2054

2055

2056

2057

2058

2059

2060

2061

2062

2063

2064

2065

2066

2067

2068

2069

2070

2071

2072

2073

2074

2075

2076

2077

2078

2079

2080

2081

2082 
Supplementary Table 4. Characteristics of Bacteria Isolated in the Different Infections Diagnosed at Enrollment in Both Treatment Arms

\begin{tabular}{|c|c|c|c|}
\hline Characteristic & $\begin{array}{l}\text { Albumin plus antibiotics } \\
\qquad(\mathrm{n}=61)\end{array}$ & $\begin{array}{c}\text { Antibiotics } \\
\text { alone } \\
(n=57)\end{array}$ & Total \\
\hline Number of isolated bacteria, $\mathrm{n}$ & 35 & 34 & 69 \\
\hline Number of gram-negative bacteria & 23 & 19 & 42 \\
\hline \multicolumn{4}{|l|}{ Number of each isolate } \\
\hline Escherichia coli & 13 & 11 & 24 \\
\hline ESBL & 5 & 4 & \\
\hline Carbapenem-resistant & & 1 & \\
\hline Klebsiella pneumoniae & 4 & 3 & 7 \\
\hline ESBL & & 1 & \\
\hline Carbapenem-resistant & & 1 & \\
\hline Klebsiella oxytoca & 1 & & 1 \\
\hline Enterobacter spp & & 3 & 3 \\
\hline Citrobacter spp & 1 & 1 & 2 \\
\hline Proteus spp & 1 & & 1 \\
\hline Pseudomonas aeruginosa & 1 & & 1 \\
\hline $\begin{array}{l}\text { Stenotrophomonas } \\
\text { maltophilia }\end{array}$ & 1 & & 1 \\
\hline Acinetobacter baumanii & & 1 & 1 \\
\hline Bacteroides fragilis & 1 & & 1 \\
\hline Number of gram-positive cocci & 10 & 13 & 23 \\
\hline \multicolumn{4}{|l|}{ Number of each isolate } \\
\hline Enterococcus faecalis & 2 & 1 & 3 \\
\hline Staphylococcus aureus & 4 & 6 & 10 \\
\hline Methicillin-sensitive $S$ aureus & 2 & 4 & \\
\hline Methicillin-resistant $S$ aureus & 2 & 2 & \\
\hline Enterococcus faecium (VSE) & 1 & & 1 \\
\hline Streptococcus pneumoniae & & 1 & 1 \\
\hline Streptococcus viridans & & 3 & 3 \\
\hline Streptococcus agalactiae & 2 & & 2 \\
\hline Streptococcus oralis & 1 & 1 & 2 \\
\hline Other GPC & & 1 & 1 \\
\hline Number of other organisms (n) & 2 & 2 & 4 \\
\hline Haemophilus influenza & - & 2 & 2 \\
\hline Clostridium difficile & 2 & - & 2 \\
\hline
\end{tabular}

ESBL, extended-spectrum $\beta$-lactamase; GPC, an घ; VSE, vancomycin-susceptible Enterococcus. 
Supplementary Table 5. Baseline Characteristics of the Per-Protocol Population

2263

2264

2265

2266

2267

2268

Demographic, clinical, and laboratory data

Age, $y$

Male sex, $\mathrm{n}(\%)$

Alcoholic cirrhosis, $\mathrm{n}(\%)$

Diabetes mellitus, $\mathrm{n}(\%)$

Ascites, $\mathrm{n}(\%)$

Mean arterial pressure, $\mathrm{mm} \mathrm{Hg}$

Median value (interquartile range) for serum bilirubin, $\mathrm{mg} / \mathrm{dL}$

Serum albumin, $g / L$

Prothrombin ratio, $\mathrm{n}(\%)$

Serum creatinine, $m g / d L$

Serum sodium, $\mathrm{mEq} / \mathrm{L}$

Serum bilirubin $\geq 4 \mathrm{mg} / \mathrm{dL}, \mathrm{n}(\%)$

Serum creatinine $\geq 1.2 \mathrm{mg} / \mathrm{dL}, \mathrm{n}(\%)$

Serum sodium $<130 \mathrm{mEq} / \mathrm{L}, \mathrm{n}(\%)$

Serum creatinine $\geq 1.2 \mathrm{mg} / \mathrm{dL}$ or serum sodium $<130 \mathrm{mEq} / \mathrm{L}, \mathrm{n}(\%)$

Median values (interquartile range) for markers of inflammation and circulatory dysfunction

White blood cell count, $x 10^{9} / \mathrm{L}$

Serum C-reactive protein, $\mathrm{mg} / \mathrm{dL}$

Plasma interleukin $6, \mathrm{pg} / \mathrm{mL}$

Plasma renin concentration, $\mu / U / m L$

Presence of ACLF or kidney dysfunction, $n(\%)$

ACLF-1

ACLF-2

ACLF

Kidney dysfunction

ACLF or kidney dysfunction

Liver scores

Child-Pugh score, points

Model of End-Stage Liver Disease score, points

CLIF-C OF score, points

Site and severity of infection, $\mathrm{n}(\%)$

Pneumonia

Urinary tract infection

Spontaneous or secondary bacteremia

Cellulitis

Cholangitis

Unproven infections

Other

Presence of sepsis, $\mathrm{n}(\%)$

Site of acquisition of infection, $n(\%)$

Community-acquired

Health care-associated

Nosocomial

Results of microbial culture, $\mathrm{n}(\%)$

Culture-positive

Presence of multidrug-resistant bacteria
Antibiotics
alone

antibiotics

$(n=56)$

$(\mathrm{n}=51)$

$57.6 \pm 10.7$

$33(64.7)$

$36(70.6)$

$17(33.3)$

$38(74.5)$

$79.1 \pm 10.7$

$4.6(2.0-11.0)$

$77.5 \pm 11.4$

$26.1 \pm 6.6$

49.8 (12.7)

$1.3 \pm 0.7$

$131.6(6.3)$

31 (56.4)

31 (56.4)

30 (53.6)

45 (80.4)

$26.4 \pm 5.8$

$48.5 \pm 16.3$

$1.1(0.4)$

$132.4(5.4)$

$30(58.8)$

$24(47.1)$

$25(49.0)$

$39(76.5)$

$7.7(4.4-11.7)$

$7.1(4.8-11.5)$

$11.9(4.8-48.0)$

38 (22-159)

242 (46-903)

11 (19.6)

$3(5.4)$

$14(25.0)$

10 (17.9)

24 (42.8)

$10.3 \pm 2.1$

$19.5 \pm 5.6$

$7.3 \pm 1.6$

14 (25.0)

15 (26.8)

11 (19.6)

2 (3.6)

3 (5.4)

9 (16.1)

2 (3.6)

$32(57.1)$

16 (28.6)

$36(64.3)$

$4(7.1)$

31 (55.4)

$10(17.9)$
$15.0(4.1-34.2)$

38 (18-71)

93 (26-327)

$6(11.8)$

1 (1.9)

7 (13.7)

5 (9.8)

12 (23.5)

$10.4 \pm 1.9$

$19.4 \pm 5.1$

$7.5 \pm 1.7$

20 (39.2)

10 (19.6)

8 (15.7)

$5(9.8)$

0

8 (15.7)

0

25 (49.0)

14 (27.5)

$34(66.7)$

3 (5.9)

29 (56.9)

14 (27.5)
$P$ value

.071

.067

.003

.090

.062

.046

.051

.085

.071

.006

.051

.080

.034

.064

.063

.090

.091

.059

.018

.069

.014

.023

.003

.076

.095

.063

.011

.038

.059

.019

.024

.096

.050

.040

.095

.

.088

.024
2269

2270

2271

2272

2273

2274

2275

2276

2277

2278

2279

2280

2281

2282

2283

2284

2285

2286

2287

2288

2289

2290

2291

2292

2293

2294

2295

2296

2297

2298

2299

2300

2301

2302

2303

2304

2305

2306

2307

2308

2309

2310

2311

2312

2313

2314

2315

2316

2317

2318 
Supplementary Table 7. New Events During Hospitalization and Short-Term Mortality in the Per-Protocol Population

\begin{tabular}{cccc}
\hline & Albumin plus & Antibiotics & \\
Event & antibiotics $(\mathrm{n}=56)$ & alone $(\mathrm{n}=51)$ & $P$ value \\
\hline
\end{tabular}

New bacterial infections

Patients with infections, $\mathrm{n}(\%)$

Patients with proven or unproven infections

Patients with proven infections

Type and severity of infections, $\mathrm{n}(\%)$

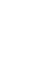

Pneumonia

Spontaneous or secondary bacteremia

Urinary tract infection

Spontaneous bacterial peritonitis

Cellulitis

Unproven infections

Clostridium difficile infection

Other

Number of infectious episodes

Presence of sepsis

Microbiology and course of infection, $n$ (\% of infections)

Culture positive ${ }^{a}$

Multidrug-resistant bacteria

Adequate empirical antibiotics

Septic shock

Infection resolution

New episodes of ACLF, $n(\%)$

Patients with ACLF, $\mathrm{n}(\%)$

ACLF-1

ACLF-2

ACLF-3

Overall ACLF

Potential mechanisms of the new ACLF, $\mathrm{n}(\%)$

Recurrence of a resolved ACLF

Precipitated by baseline bacterial infection ${ }^{b}$

Precipitated by new bacterial infection

Unknown mechanism

Course of ACLF, n (\%)

Resolution

Other clinical events, $\mathrm{n}(\%)$

Variceal bleeding

Nonvariceal bleeding

Atrial fibrillation

Pulmonary edema

Liver transplantation and mortality, $\mathrm{n}(\%)$

Liver transplantation during hospitalization

Liver transplantation by $90 \mathrm{~d}$

Hospital mortality

90-d mortality ${ }^{c}$

$11(21.6)$

11 (21.6)

13

$4(7.1)$

6

$1(1.8)$

$1(1.8)$

$1(1.8)$

$1(1.8)$

$1(1.8)$

$1(1.8)$

0

$4(7.1)$

0

$3(5.9)$

$4(7.8)$

$3(5.9)$

0

0

0

$2(3.9)$

$3(5.9)$

$1(1.9)$

1 (16.7)

$1(14.3)$

$4(66.7)$

2 (33.3)

$4(66.7)$

6 (10.7)

4 (7.1)

0

10 (17.9)

1 (1.8)

$4(7.1)$

$1(1.8)$

4 (7.1)

$9(69.2)$

6 (46.2)

11 (84.6)

$3(23.1)$

$10(76.9)$

3 (5.4)

4 (7.1)

$4(7.1)$

2 (3.6)

1 (1.8)

1 (1.8)

$2(3.6)$

6 (10.7)

15 (26.8)

NOTE. Categorical variables as number and percentage or number.

ACLF, acute-on-chronic liver failure; ESBL, extended-spectrum $\beta$-lactamase.

${ }^{a}$ In the albumin-plus-antibiotics group there was 1 infection caused by ESBL Escherichia coli. In the antibiotics-alone group there were 3 infections caused by

${ }^{b}$ In these cases, ACLF developed during the next 7 days after inclusion with no new infections or other clinical events within this period. 
Supplementary Table 8. Analysis of Risk Factors at Inclusion for the Development of New Bacterial Infections, New ACLF, Pulmonary Edema, and Short-Term Mortality

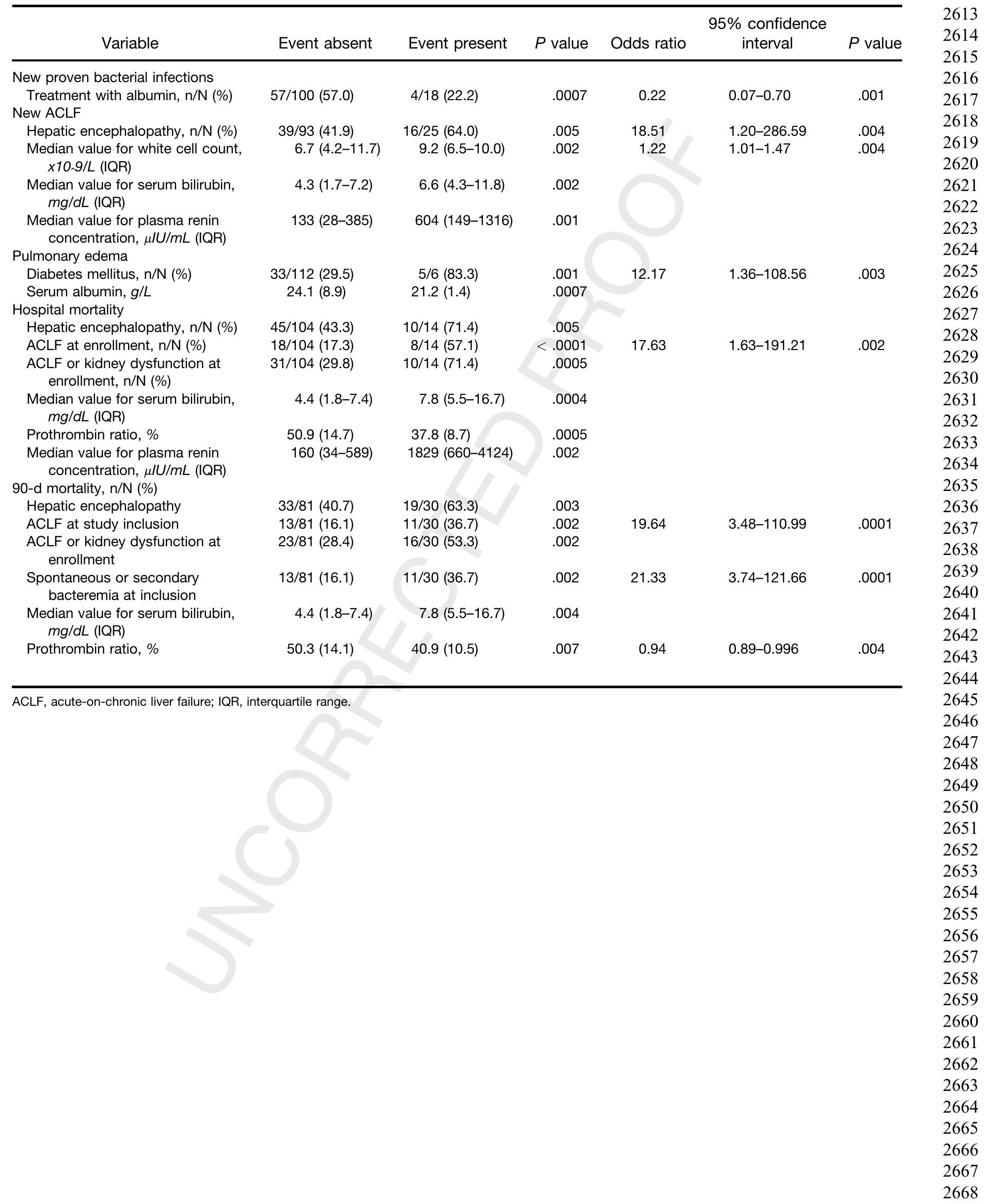




\section{1.e13 Fernández et al}

Clinical Gastroenterology and Hepatology Vol. —, No.

Supplementary Table 9. Correlation Between Baseline Serum Albumin Levels and the Response to Albumin Treatment in Patients Included in the AlbuminPlus-Antibiotics Group

Baseline serum

albumin level, $g / L \quad P$ value

Resolution of baseline ACLF

No

$26.9(5.8)$

Yes

$25.6(7.5)$

.06527

ACLF onset during hospitalization

No

Yes

$26.2(5.4)$

$24.9(7.2)$

.03697

$26.3(6.1)$

$24.1(5.6)$

.01481

Correlation with changes from baseline mean arterial pressure At $3 \mathrm{~d}$

At $7 \mathrm{~d}$

At $3 / 7 \mathrm{~d}$

$-0.036 \quad .07152$

$0.160 \quad .02992$

$-0.019 \quad .08469$

Correlation with changes from baseline interleukin 6

At $3 \mathrm{~d}$

At $7 \mathrm{~d}$

At $3 / 7 d$

0.043

$-0.015$

0.025

.06932

ACLF, acute-on-chronic liver failure. 
Supplementary Table 10. Main Events Occurring During Hospitalization in Patients Who Died and Causes of Death

\begin{tabular}{|c|c|c|c|c|c|c|c|c|c|}
\hline $\begin{array}{l}\text { Patient } \\
\text { ID }\end{array}$ & $\begin{array}{l}\text { Adequate } \\
\text { empirical } \\
\text { antibiotics }\end{array}$ & $\begin{array}{l}\text { Resolution of } \\
\text { baseline } \\
\text { infection }\end{array}$ & $\begin{array}{l}\text { ACLF at } \\
\text { enrollment }\end{array}$ & $\begin{array}{c}\text { Resolution of } \\
\text { ACLF at } \\
\text { enrollment }\end{array}$ & $\begin{array}{l}\text { New proven } \\
\text { bacterial } \\
\text { infection/day/ } \\
\text { resolution }\end{array}$ & $\begin{array}{c}\text { New ACLF/ } \\
\text { day }\end{array}$ & $\begin{array}{l}\text { Final ACLF } \\
\text { grade }\end{array}$ & $\begin{array}{l}\text { Day of death } \\
\text { since } \\
\text { enrollment }\end{array}$ & Cause of death \\
\hline \multicolumn{10}{|c|}{ Antibiotics-alone group $(n=6)$} \\
\hline $1_{(02-08)}$ & Yes & No & Yes & No & No/NA/NA & No/NA & ACLF-3 & Day 13 & $\begin{array}{l}\text { Multiorgan failure; no } \\
\text { identifiable trigger }\end{array}$ \\
\hline $2_{(03-08)}$ & No & Yes & No & - & Yes/Day 12/No & Yes/Day 7 & ACLF-3 & Day 26 & Pulmonary edema \\
\hline $3_{(14-01)}$ & No & Yes & Yes & No & No/NA/NA & No/NA & ACLF-3 & Day 28 & $\begin{array}{l}\text { Multiorgan failure; no } \\
\text { identifiable trigger }\end{array}$ \\
\hline $4_{(17-02)}$ & Yes & Yes & Yes & Yes & $\begin{array}{l}\text { Yes/Day 3/Yes } \\
\text { Yes/Day 50/No }\end{array}$ & Yes/Day 55 & ACLF-3 & Day 56 & Septic shock \\
\hline $5(21-03)$ & Yes & Yes & No & - & No/NA/NA & Yes/Day 21 & ACLF-3 & Day 22 & $\begin{array}{l}\text { Multiorgan failure; no } \\
\text { identifiable trigger }\end{array}$ \\
\hline $6_{(24-13)}$ & Yes & No & Yes & No & No/NA/NA & No/NA & ACLF-3 & Day 13 & Septic shock \\
\hline \multicolumn{10}{|c|}{ Albumin-plus-antibiotics group $(n=8)$} \\
\hline $1_{(02-07)}$ & Yes & Yes & Yes & Yes & $\begin{array}{l}\text { Yes/Day 9/Yes } \\
\text { Yes/Day 21/No }\end{array}$ & Yes/Day 10 & ACLF-3 & Day 22 & $\begin{array}{l}\text { Hypovolemic shock; } \\
\text { variceal hemorrhage }\end{array}$ \\
\hline $2_{(06-01)}$ & No & No & Yes & No & No/NA/NA & No/NA & ACLF-3 & Day 5 & Septic shock \\
\hline $3_{(09-08)}$ & Yes & Yes & Yes & Yes & No & Yes/Day 28 & ACLF-3 & Day 31 & $\begin{array}{l}\text { Multiorgan failure without } \\
\text { identifiable trigger }\end{array}$ \\
\hline $4_{(12-02)}$ & Yes & No & No & - & No/NA/NA & Yes/Day 3 & ACLF-3 & Day 8 & $\begin{array}{l}\text { Multiorgan failure without } \\
\text { identifiable trigger }\end{array}$ \\
\hline $5_{(14-15)}$ & Yes & No & Yes & Yes & No/NA/NA & Yes/Day 12 & ACLF-3 & Day 12 & $\begin{array}{l}\text { Multiorgan failure without } \\
\text { identifiable trigger }\end{array}$ \\
\hline $6_{(17-08)}$ & No & No & No & - & No/NA/NA & Yes/Day 3 & ACLF-3 & Day 6 & $\begin{array}{l}\text { Multiorgan failure without } \\
\text { identifiable trigger }\end{array}$ \\
\hline $7_{(23-02)}$ & Yes & No & No & - & No/NA/NA & Yes/Day 3 & ACLF-2 & Day 7 & $\begin{array}{l}\text { Multiorgan failure without } \\
\text { identifiable trigger }\end{array}$ \\
\hline $8_{(24-05)}$ & Yes & No & No & - & No/NA/NA & Yes/Day 3 & ACLF-2 & Day 16 & $\begin{array}{l}\text { Multiorgan failure without } \\
\text { identifiable trigger }\end{array}$ \\
\hline
\end{tabular}

